\title{
A Parallel External Memory System
}

\author{
By \\ Mohammad Reza Nikseresht
}

\author{
A thesis submitted to \\ the Faculty of Graduate Studies and Research \\ in partial fulfilment of \\ the requirements for the degree of \\ Master of Computer Science \\ Ottawa-Carleton Institute for Computer Science \\ School of Computer Science \\ Carleton University \\ Ottawa, Ontario
}

August 28, 2007

(C) Copyright

2007, Mohammad Reza Nikseresht 


$\begin{array}{ll}\begin{array}{l}\text { Library and } \\ \text { Archives Canada }\end{array} & \begin{array}{l}\text { Bibliothèque et } \\ \text { Archives Canada }\end{array} \\ \begin{array}{l}\text { Published Heritage } \\ \text { Branch }\end{array} & \begin{array}{l}\text { Direction du } \\ \text { Patrimoine de l'édition }\end{array} \\ \begin{array}{l}\text { 395 Wellington Street } \\ \text { Ottawa ON K1A ON4 }\end{array} & \begin{array}{l}\text { 395, rue Wellington } \\ \text { Ottawa ON K1A ON4 } \\ \text { Canada }\end{array}\end{array}$

Your file Votre référence ISBN: 978-0-494-33683-0 Our file Notre référence ISBN: 978-0-494-33683-0

NOTICE:

The author has granted a nonexclusive license allowing Library and Archives Canada to reproduce, publish, archive, preserve, conserve, communicate to the public by telecommunication or on the Internet, loan, distribute and sell theses worldwide, for commercial or noncommercial purposes, in microform, paper, electronic and/or any other formats.

The author retains copyright ownership and moral rights in this thesis. Neither the thesis nor substantial extracts from it may be printed or otherwise reproduced without the author's permission.
AVIS:

L'auteur a accordé une licence non exclusive permettant à la Bibliothèque et Archives Canada de reproduire, publier, archiver, sauvegarder, conserver, transmettre au public par télécommunication ou par l'Internet, prêter, distribuer et vendre des thèses partout dans le monde, à des fins commerciales ou autres, sur support microforme, papier, électronique et/ou autres formats.

L'auteur conserve la propriété du droit d'auteur et des droits moraux qui protège cette thèse. $\mathrm{Ni}$ la thèse ni des extraits substantiels de celle-ci ne doivent être imprimés ou autrement reproduits sans son autorisation.
In compliance with the Canadian

Privacy Act some supporting forms may have been removed from this thesis.

While these forms may be included in the document page count, their removal does not represent any loss of content from the thesis.
Conformément à la loi canadienne sur la protection de la vie privée, quelques formulaires secondaires ont été enlevés de cette thèse.

Bien que ces formulaires aient inclus dans la pagination, il n'y aura aucun contenu manquant.

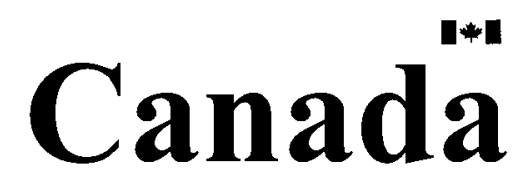




\begin{abstract}
In this work we describe a Parallel External Memory System (PEMS) which provides a framework and interface to convert Bulk Synchronous Parallel (BSP) programs into external memory programs based on the theoretical results of $[12,30]$. The results in $[12,30]$ show how to adapt parallel algorithms designed for a large number of processors without disks to smaller, realistic numbers of processors, each with its own disk system. While the theory shows that for selected problems this can be done with the same amount of $\mathrm{I} / \mathrm{O}$, asymptotically, as an single processor algorithm, it has not been clear whether this approach is attractive in practice. This work takes a step forward in this regard, showing timing results for sorting and list ranking that are comparable with TPIE and STXXL. Our experiments show that this approach is practical and promising and the run times scale predictable with the number of processors and with the problem size.
\end{abstract}




\section{Acknowledgments}

I would like to thank my supervisors David Hutchinson and Anil Maheshwari for their help, attention and advice during this work. It was not possible to finish this work without their help.

I wish to thank Anil Maheshwari for his patience and time discussing theoretical aspects of this work and his help writing our paper. I would also like to thank him for his financial support. I would like to thank David Hutchinson for his time discussing theoretical and technical issues, his help writing our paper and his many comments on my thesis which makes my writing readable.

I would like to thank Ryan Taylor who was a great help during the experiments on HPCVL machines by providing a dedicated special configuration of machines for our tests.

I would also like the staff at school of computer science for the great working environment during my studies. Special thanks to Linda Pfeiffer and Claire Ryan.

Finally, I must acknowledge the support and help from family members. Thanks to my parents who have always been supportive of stretching for new and higher levels of science. My very special thanks to my wife Arezoo and our son Ali who have been patient and supportive during this period of my studies. It was not possible to continue without your help and support. 


\section{Contents}

1 Introduction 1

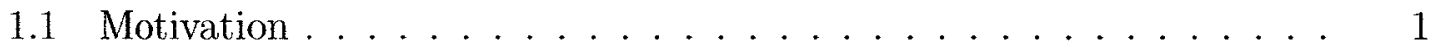

1.2 Model of Computation . . . . . . . . . . . . . 2

1.2.1 Parallel Disk Model (PDM) . . . . . . . . . . 2

1.2.2 The Bulk Synchronous Parallel Model (BSP) . . . . . . . . . 3

1.2.3 The Coarse Grained Multicomputer (CGM) Model . . . . . . 5

1.3 Overview of the Results ................. 5

1.4 Organization of the Thesis .................. 6

$\begin{array}{lll}2 & \text { Related Work } & 7\end{array}$

2.1 External Memory (EM) Algorithms . . . . . . . . . . . 7

2.2 Techniques for Designing EM Algorithms . . . . . . . . . . 8

2.2 .1 Disk Striping . . . . . . . . . . . . . . . . 9

2.2 .2 Distribution . . . . . . . . . . . . . . 9

2.2 .3 Merge ......................... 10

2.2.4 Distribution Sweeping . . . . . . . . . . 10

2.2.5 Time-forward processing . . . . . . . . . . 10

2.2.6 Techniques Based on Simulation of Parallel Algorithms . . . . 12

ii 
2.3 Some External Memory Algorithms . . . . . . . . . . . . . . . . . 13

2.3 .1 Sorting . . . . . . . . . . . . . . . 13

2.3 .2 List Ranking . . . . . . . . . . . . . . . . . . 14

2.4 Implementation Studies $\ldots \ldots \ldots \ldots \ldots$

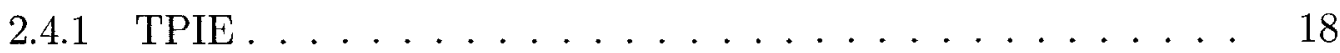

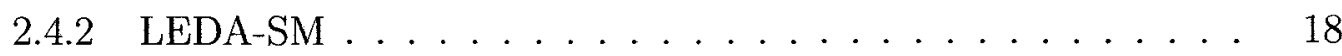

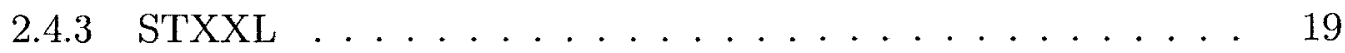

$2.4 .4 \quad$ SSCRAP . . . . . . . . . . . . . . . . 20

2.4.5 Previous Simulation Implementation Work . . . . . . . . . 21

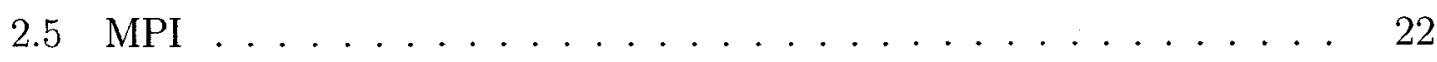

3 BSP Algorithms in External Memory 24

3.1 Single Processor Simulation Algorithm . . . . . . . . . . . 24

3.2 Multiprocessor Simulation Algorithm . . . . . . . . . . 25

3.3 Personalized Communication . . . . . . . . . . . . . . 27

4 Software Design $\quad 29$

4.1 Introduction . . . . . . . . . . . . . . . . . . . . . . . 29

4.1 .1 Practical Considerations . . . . . . . . . . . . . 32

4.2 Virtual processor simulation and process management . . . . . . 35

4.3 Context Switching and Memory Management $\ldots \ldots \ldots \ldots$

4.4 Disk I/O Subsystem . . . . . . . . . . . . . . 38

4.5 PEMS System Interface and Messaging . . . . . . . . . 38

$4.5 .1 \quad$ EM_Allgather . . . . . . . . . . . . . . . . 40

$4.5 .2 \quad$ EM_Alltoallv . . . . . . . . . . . . . . . . 42

iii 
4.5.3 Direct delivery to context .............. 46

4.6 Discussion on Design Choices . . . . . . . . . . . . 46

4.6.1 Representation of virtual processors . . . . . . . . . . . 46

4.6.2 Choices for Communication . . . . . . . . . . . 47

4.6.2.1 A Discussion on Asynchronous/Direct Network Communication ................... 49

4.6.3 Direct and Asynchronous I/O ............ 50

4.6.4 Kernel Level Threads Versus User Level Threads . . . . . . . 52

5 Experiments $\quad 53$

5.1 Experimental Setup . . . . . . . . . . . . . . 54

5.2 Testing EM_Alltoallv . . . . . . . . . . . . . . 54

5.2.1 Single Processor Experiments with EM_Alltoallv . . . . . . 55

5.2 .2 Multiple Processor Tests . . . . . . . . . . . 57

5.3 Parallel Sample Sort . . . . . . . . . . . . . 58

5.3.1 Experimental Results with Parallel Sample Sort . . . . . . . 59

5.3.1.1 Single Processor Experiments . . . . . . . . 59

5.3.1.2 Multiple Processor Experiments ......... 61

5.4 Randomized Parallel List Ranking . . . . . . . . . . . . . . . . 64

5.4.1 Experimental Results with List Ranking . . . . . . . . . 65

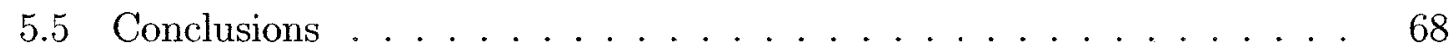

6 Concluding Remarks And Future Work $\quad 69$

$\begin{array}{ll}\text { Appendices } & 74\end{array}$

iv 
A System Configurations for Experiments

A.1 Machine Configuration A for Single Processor Tests . . . . . . . . . . 74

A.2 Cluster Configuration B for Multiple Machine Experiments . . . . . . 75

A.3 Cluster Configuration C for Multiple Machine Experiments . . . . 75

$\begin{array}{ll}\text { Bibliography } & 77\end{array}$ 


\section{List of Tables}

2.1 Fundamental EM operations . . . . . . . . . . . . . . 9

4.1 Advantages and disadvantages of kernel and user level threads . . . 52 


\section{List of Figures}

1.1 A BSP computation divided into supersteps (from [30]). . . . . . . 4

2.1 The circuit evaluation problem. . . . . . . . . . . . 11

2.2 Illustration of merge sort with $\frac{N}{M}=6$ and $R=3$ and $2 \ldots \ldots$

2.3 An example showing how List Ranking Algorithm 2.1 works . . . . . 16

2.4 STXXL System Software Layers (from [16]) . . . . . . . . . . . . 19

4.1 A simple MPI program . . . . . . . . . . . . . . 30

4.2 MPI program in Figure 4.1 converted to PEMS program . . . . . . . 31

4.3 PEMS System Software Layers . . . . . . . . . . . . . . . 32

4.4 PEMS on $p$ real processors and 3 threads per processor. . . . . . . 35

4.5 Balanced routing bins and bin counters in Alltoallv . . . . . . . . 43

5.1 Wall clock timings for EM_Alltoallv on a single processor. The $\mathrm{X}$-axis is the problem size in millions of integers. The $\mathrm{Y}$-axis is wall clock time in seconds. ............................ 55

5.2 Wall clock timings of EM_Alltoallv for data sizes of 500, 1000 and 1500 million integers. The $\mathrm{X}$-axis is the number of virtual processors. The $\mathrm{Y}$-axis is wall clock time in seconds. . . . . . . . . . . .

vii 
5.3 Wall clock timings of EM_Alltoallv for $p=2,4$ and 8. The $\mathrm{X}$-axis is the problem size in millions of integers. The Y-axis is wall clock time in

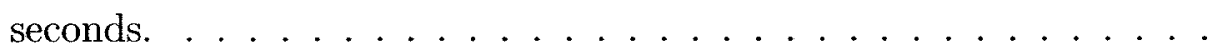

5.4 Normalized wall clock timings of EM_Alltoallv for data sizes of 200, 500 and 800 million integers. . . . . . . . . . . . 58

5.5 Single processor results, $\mathrm{X}$-axis is the problem size in millions of integers. The $\mathrm{Y}$-axis is the wall clock time in seconds. . . . . . . . 60

5.6 Normalized wall clock timings for sorting. The $\mathrm{X}$-axis is the problem size in billions of integers. The Y-axis is wall clock time per data item in microseconds. . . . . . . . . . . . . . . .

5.7 Wall clock timings for sorting. The $\mathrm{X}$-axis is the problem size in billions of integers. The $\mathrm{Y}$-axis is wall clock time in seconds. . . . . . . 62

5.8 Speedup of PEMS Sample Sort. . . . . . . . . . . . . 63

5.9 Normalized wall clock timing of list ranking on $1,2,3,4,5$ and 6 real processors. . ..................... 66

5.10 Wall clock timing of list ranking on $1,2,3,4,5$ and 6 real processors. . . 67

5.11 Speedup of PEMS list ranking as number of real processors increase. The $\mathrm{X}$-axis is the number of real processors. The $\mathrm{Y}$-axis is wall clock time in seconds. . . . . . . . . . . . . . . . . 


\section{Chapter 1}

\section{Introduction}

\subsection{Motivation}

Although recently there have been dramatic increases in computer resources including computing power, memory size and disk size, applications also have grown in terms of their demands on computer resources. Many scientific and commercial applications require storage and processing of huge amount of data. These include applications in geographical information systems, astrophysics, computational biology, VLSI design, weather prediction and billing systems of telecommunication companies [29]. The size of data in these applications is in the order of hundreds of gigabytes to terabytes. Such huge data sets do not generally fit into main memories, and so the data sets must reside on disks. Unfortunately, disk speeds have not increased as much as main memory speeds. A disk access may be $10^{6}$ times slower than a main memory due to the mechanical characteristics of the hard disks. Data transfer between main memory and external memory is a bottleneck and limits the size of very large scale applications. To overcome this problem, locality in the pattern of an application's 
memory references is exploited. Often some parts of the data are referenced frequently for a period of time and then the program changes attention to some other part of the data. Such sets of data usually are called "working sets" [17]. Operating systems track the working sets and typically use heuristic techniques to pre-fetch them into main memory before the application tries to access the data, so the total number of I/O transfers is reduced. But these techniques may not take full advantage of the potential for locality in every program. So, taking advantage of locality of references from within the algorithms was considered, and a new class of algorithms specifically designed for huge data sets emerged. These algorithms are called External Memory (EM) algorithms or I/O efficient algorithms.

\subsection{Model of Computation}

In this section we discuss briefly the models of computation which are needed to understand this work, the Parallel Disk Model (PDM) [51, 52], the Bulk Synchronous Parallel Model (BSP) [47] and the Coarse Grained Multicomputer (CGM) [11, 40].

\subsubsection{Parallel Disk Model (PDM)}

The most widely used model for designing EM algorithms is the Parallel Disk Model by Vitter and Shriver $[51,52]$. This model assumes that it is possible to access D disks simultaneously in a single I/O operation. It allows the disks to be attached to a single processor or to multiple processors. These multiple processors are then interconnected through some sort of network. In this model the $\mathrm{I} / \mathrm{O}$ is handled directly by the application. It tries to transfer DB items to/from the disks simultaneously 
in every $\mathrm{I} / \mathrm{O}$ operation if possible. The I/O complexity is measured by counting the number of I/O operations needed by a problem $\mathcal{P}$ as the problem size $\mathrm{N}$ approaches infinity. Other costs are ignored. Other assumptions and parameters in this model are as follows: $\mathrm{M}$ is the size of internal memory available to the algorithm (meaning that it can hold $\mathrm{M}$ data items). It is also assumed that for each $\mathrm{I} / \mathrm{O}$ operation one block of data for each of the disks can be transferred and each block contains B items. The parameters in this model are:

$N$ : Total number of data items in the problem (problem size).

$B$ : Disk block size.

$D:$ Total number of disks used.

$M:$ Size of the available internal memory.

\subsubsection{The Bulk Synchronous Parallel Model (BSP)}

The BSP model proposed by Valiant [47] is a "bridging model for parallel computation". It is possible for both hardware and software designs to agree on this model. As it is neither a hardware model nor a programming model for parallel computers but something in between, it can be useful for hardware designers, algorithm designers and programmers at the same time. In this model a number of independent processors collaborate with each other to solve a problem. They can send and receive messages to each other through a network or any other communication system. Based on this model a parallel computation proceeds as a series of supersteps separated by synchronizations. These supersteps can be further divided into computation supersteps 


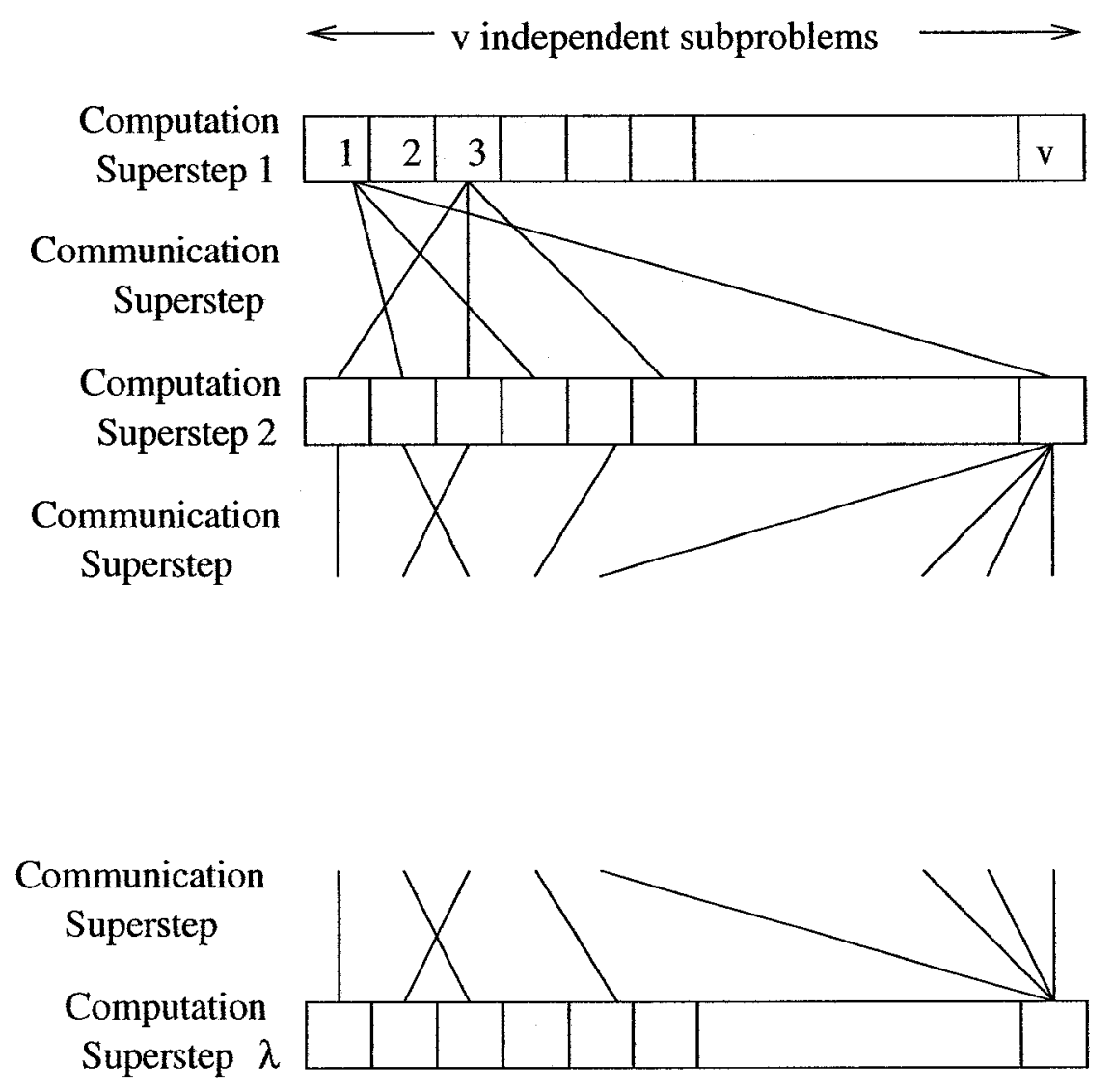

Figure 1.1: A BSP computation divided into supersteps(from [30]).

and communication supersteps. Figure 1.1 illustrates this concept. If, in a communication superstep each processor sends and receives $O(h)$ data, such a superstep is called an $h$-relation. The cost of computation on this model is represented by the sum of computation time, communication time and synchronization time. The following parameters are defined in this model:

$N:$ is the number of items in the problem to be solved.

$v$ : is the number of available processors. We use $v$ because in our system the processors in a BSP algorithm are typically viewed as virtual processors simulated on one or more real machines. 
$g:$ is the time required to send a simple word of data between two processors. This time is measured in number of CPU operations.

$L:$ is the minimum latency or the setup time of a superstep which is measured in number of CPU operations.

\subsubsection{The Coarse Grained Multicomputer (CGM) Model}

The CGM model $[11,40]$ is a special case of the BSP model. In this model it is assumed that at the beginning of computation, each of $v$ processors contains $O\left(\frac{N}{v}\right)$ data items. In each computation superstep, the model assumes that each processor performs computation internally on $O(N / v)$ of data and in each communication superstep each processor sends and/or receives $O\left(\frac{N}{v}\right)$ items of data, so in the CGM model $h=O\left(\frac{N}{v}\right)$ for every superstep. This assumption on the communication volume differentiates the CGM model from the general BSP model.

\subsection{Overview of the Results}

In this work we develop and test a system for writing Parallel External Memory programs. Using the approach of $[10,12,13,30]$, we convert an implementation of an optimal parallel algorithm designed for a large number of processors without disks to smaller, realistic numbers of processors, each with its own disk system. While the theory shows that for selected problems this can result in a parallel EM algorithm that uses the same amount of I/O, asymptotically, as an optimal EM algorithm, it has not been clear whether this approach is attractive in practice. This work takes a step forward in this regard, showing timing results for sorting and list-ranking that 
are comparable with TPIE and STXXL, two contemporary workbenches for high performance $\mathrm{I} / \mathrm{O}$ experiments. We compare single processor instances of sorting on these workbenches with sorting using various numbers of real processors. We also performed corresponding experiments with a randomized list ranking algorithm, and report our timing results. Our experiments show that this approach is practical and promising and the run times scale predictable with the number of processors and with the problem size.

Part of this work will be presented in the $14^{\text {th }}$ International Conference on High Performance Computing [36].

\subsection{Organization of the Thesis}

In Chapter 2 we review selected topics on External Memory algorithms and previous implementation projects. In Chapter 3 we review the main theoretical idea of simulation of BSP algorithms as External Memory algorithms in more detail. In Chapter 4 we describe the internal structure of PEMS and discuss design choices that we encountered. Chapter 5 presents our experimental results with sorting and list-ranking algorithms. Chapter 6 contains concluding remarks and suggestions for further expansion of this work. 


\section{Chapter 2}

\section{Related Work}

\subsection{External Memory (EM) Algorithms}

Algorithms developed originally for internal memory models, such as the RAM and PRAM models, are frequently found to be inefficient in the EM environment. Such algorithms typically have poor "locality of reference", causing too many I/O operations to be performed during the execution of an application. As a result, researchers have developed special algorithms optimized for EM computation. In many cases, these have been "hand-crafted", so the choices for an application developer have been somewhat limited as a result. External memory algorithms are optimized for EM computation. They are designed so that their run times scale predictably even as the size of their data increases far beyond the size of internal random access memory (RAM). This huge data size requires that such algorithms optimize the transfer of data between RAM and secondary memory devices, typically moveable-head disk drives. In addition, the time to set up the data transfer (disk head movement, rotational delay) is much larger than the time to actually transfer the data. External 
memory algorithms try to group data items into blocks and access them block-wise in order to amortize the setup time over a large number of data items.

\subsection{Techniques for Designing EM Algorithms}

During the last years many techniques have been developed to exploit locality and therefore reducing I/O complexity of External Memory algorithms. The reader may refer to the survey paper [49] to get a good exposure on EM algorithms and techniques. Most of these techniques are based on the Parallel Disk Model (PDM) and focus on data structures optimized for external memory. There are a limited number of techniques $[10,12,13,30,44]$ which pay attention to the similarities between parallel algorithms and external memory algorithms. We call these, simulation techniques (they try to simulate parallel algorithms in external memory) and call other techniques, e.g. [24, 32], conventional external memory techniques. There are many conventional techniques developed to solve different problems and basically all of these techniques are different ways to reduce or optimize I/O activities. There are four conventional fundamental operations in external memory [49].

1) Scanning: Reading or writing N data items in a file.

2) Sorting: Putting the $\mathrm{N}$ items of a file in the sorted order.

3) Searching: Searching through N sorted data items in a file.

4) Outputting: Finding the $\mathrm{Z}$ answers to a query in a blocked output sensitive manner. 
Table 2.1: Fundamental EM operations

\begin{tabular}{|l|l|}
\hline Operation & I/O Bound \\
\hline Scan(N) & $\Theta\left(\frac{N}{D B}\right)$ \\
\hline Sort $(\mathrm{N})$ & $\Theta\left(\frac{N}{D B} \log _{M / B} \frac{N}{B}\right)$ \\
\hline Search(N) & $\Theta\left(\log _{D B} N\right)$ \\
\hline Output(Z) & $\Theta\left(\max \left\{1, \frac{Z}{D B}\right\}\right)$ \\
\hline
\end{tabular}

Table 2.1 lists fundamental operations with their I/O complexities based on the PDM model. Now we introduce some of the conventional EM techniques briefly.

\subsubsection{Disk Striping}

Disk striping $[32,41]$ is a technique to make $\mathrm{D}$ disks with block size $\mathrm{B}$ behave as one disk with block size DB. Then it is possible to convert an algorithm optimized for a single disk with block size BD into an optimized algorithm for D disks with block size B. Then the multiple disk algorithm will be $\Theta(D)$ times faster than the single disk algorithm. This technique can be used to get optimal multi-disk algorithms for three of the four fundamental problems. It is non-optimal for sorting [49].

\subsubsection{Distribution}

Distribution is one of the techniques to solve the sort problem. Distribution sort [33] is a recursive technique which uses $S-1$ items to partition the data into $S$ partitions or buckets. All of the elements in one bucket precede all of the elements in the next bucket. This technique recursively sorts individual buckets and then concatenates them together to create the final result. It is possible to use full or partial striping 
to utilize multiple disks [51], but full striping is not optimal. Randomized techniques have been devised for optimally using multiple disks in distribution-based algorithms $[50,31]$.

\subsubsection{Merge}

Merge is another technique which is basically used for sorting [33]. The idea is to split the data into runs that can each fit into the memory, sort these runs in the memory and write them back to the disk. Then merge these sorted runs into one final sorted run. We explain this method in more detail in Section 2.3.1.

\subsubsection{Distribution Sweeping}

Distribution sweeping[24] is a paradigm which combines the external memory distribution technique with the sweeping technique $[9,39]$ from the computational geometry to create a generalized version of the distribution technique which is capable of solving some of the computational geometry problems in external memory.

\subsubsection{Time-forward processing}

Time forward processing is a technique used in some graph related algorithms. Originally it was proposed to solve the "circuit evaluation" problem as defined in [7] and illustrated in Figure 2.1. The description of a bounded fan-in boolean circuit is stored in external memory. The problem is to evaluate the function computed by this circuit. The labels of the circuit are topologically sorted. This means that for every edge $(\mathrm{u}, \mathrm{w}), u<w$ and " $<$ " is a total order. The only assumption about the functions at each vertex is that they take at most $M / 2$ inputs. In [7] this problem is solved using 


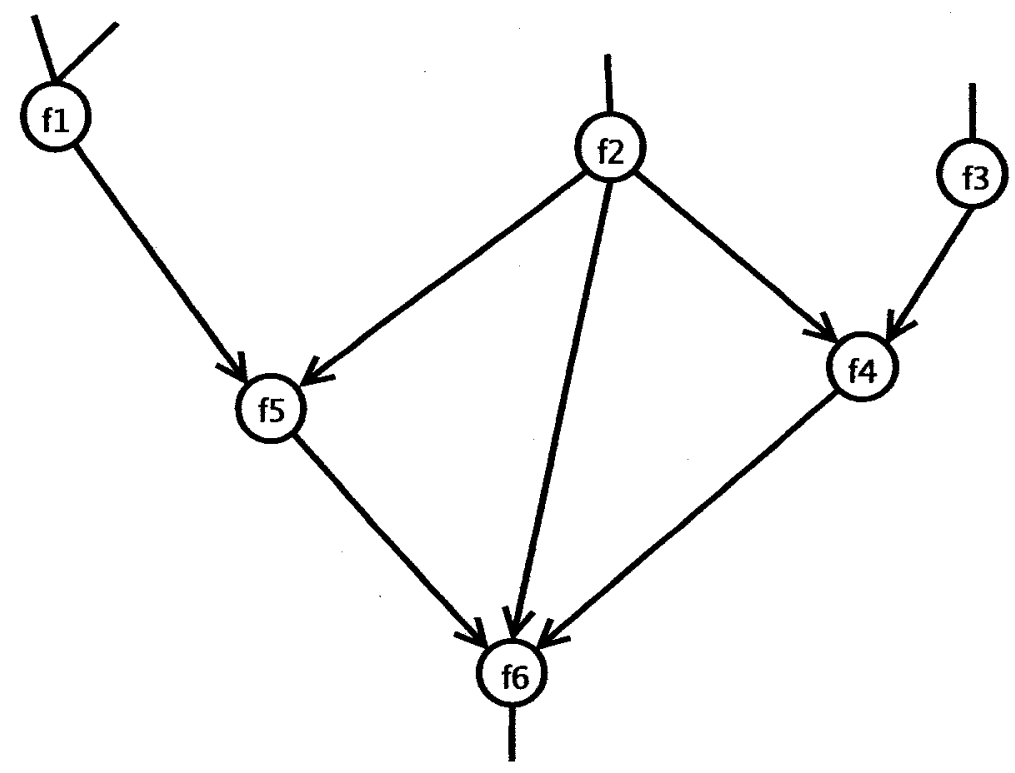

Figure 2.1: The circuit evaluation problem.

a technique that they call "time forward processing" technique. One can think of evaluating function at vertex $u$ as evaluating it at time $u$. The issue here is to ensure that at the time of evaluation of a vertex, all inputs to this function are in internal memory. Using an EM priority queue [3], when evaluating a vertex $u$ it is possible to send the function result "forward in time" to the proper vertices by inserting it into the priority queue with the priority $w$ for all edges $(u, w)$. Then performing a series of deletemin operations on the priority queue, its next elements are the inputs to the next vertex in topological order.

Theorem 2.1 [3] The I/O complexity of the time forward processing technique using priority queue is $O(\operatorname{Sort}(N))$. 


\subsubsection{Techniques Based on Simulation of Parallel Algorithms}

As we stated at the beginning of this section there is a class of EM algorithms which take advantage of similarities between EM algorithms and parallel algorithms. Chiang et al. [7] suggested that it is possible to develop an efficient EM algorithm by simulating a parallel algorithm for the same problem in external memory. Their approach involves sorting of the data between simulation steps of a PRAM algorithm to bring the data needed by a specific operation together so that the operations can be completed by simple scanning through the data. Dehne, Dittrich, Hutchinson and Maheshwari [10, 12, 13, 30] and Sibeyn and Kaufman [44] proposed a simulation approach based on the BSP model. As CGM algorithms guarantee enough locality, no sorting between simulation steps is required for such algorithms $[10,12,13,30]$.

Dehne, Dittrich, Hutchinson and Maheshwari $[10,12,13,30]$ have developed a theoretical framework for simulating BSP algorithms as EM algorithms. BSP (and therefore CGM) algorithms break a problem into subproblems on which computation can proceed independently for some period of time before synchronization, i.e. communication, is required between the processors. Between synchronization events, references to data by a particular processor are restricted to data items currently residing in the local memory of the processor. This is possible because the subproblems in a given parallel step are independent. As there are many existing efficient BSP algorithms to solve large scale problems, this framework enables us to convert these algorithms to efficient EM algorithms. Another interesting advantage of this approach is that, it is possible to run these EM algorithms on parallel machines. In fact the resulting algorithms not only are EM algorithms but also are parallel algorithms. Programs which are based on these algorithms can take advantage of both reduced 
I/O operations and the power of parallel computing on clusters of workstations or shared memory machines. This approach can be used to enable developers to convert a certain class of parallel algorithms implemented using MPI [20] to parallel external memory algorithms. We will describe this approach in more detail in Chapter 3.

\subsection{Some External Memory Algorithms}

In this section we discuss algorithms for two important and interesting problems in computer science: sorting and list ranking. Sorting is a basic problem with application in solving many different problems. List ranking has also a basic application in many graph algorithms.

\subsubsection{Sorting}

Merge sort is an example of an internal memory algorithm which is adapted for external memory. External merge sort is widely used for sorting huge data sets in external memory; for example TPIE (see section 2.4.1) has an implemention of a merge sort.

The merge sort consists of several phases. The first phase is called "run formation" phase. In this phase the "runs" of data are created. Each "run" consists of data items equal to the available memory size M. Each run is read into the memory, sorted, and is written back to the disk. Then merging phase begins. Now there are $\frac{N}{M}$ sorted runs to merge. In each merging pass, $R$ runs are merged to form a new run. This will continue until all data are merged into one sorted run. Figure 2.2 illustrates basic merge sort. Many techniques have been used to increase the performance of external 


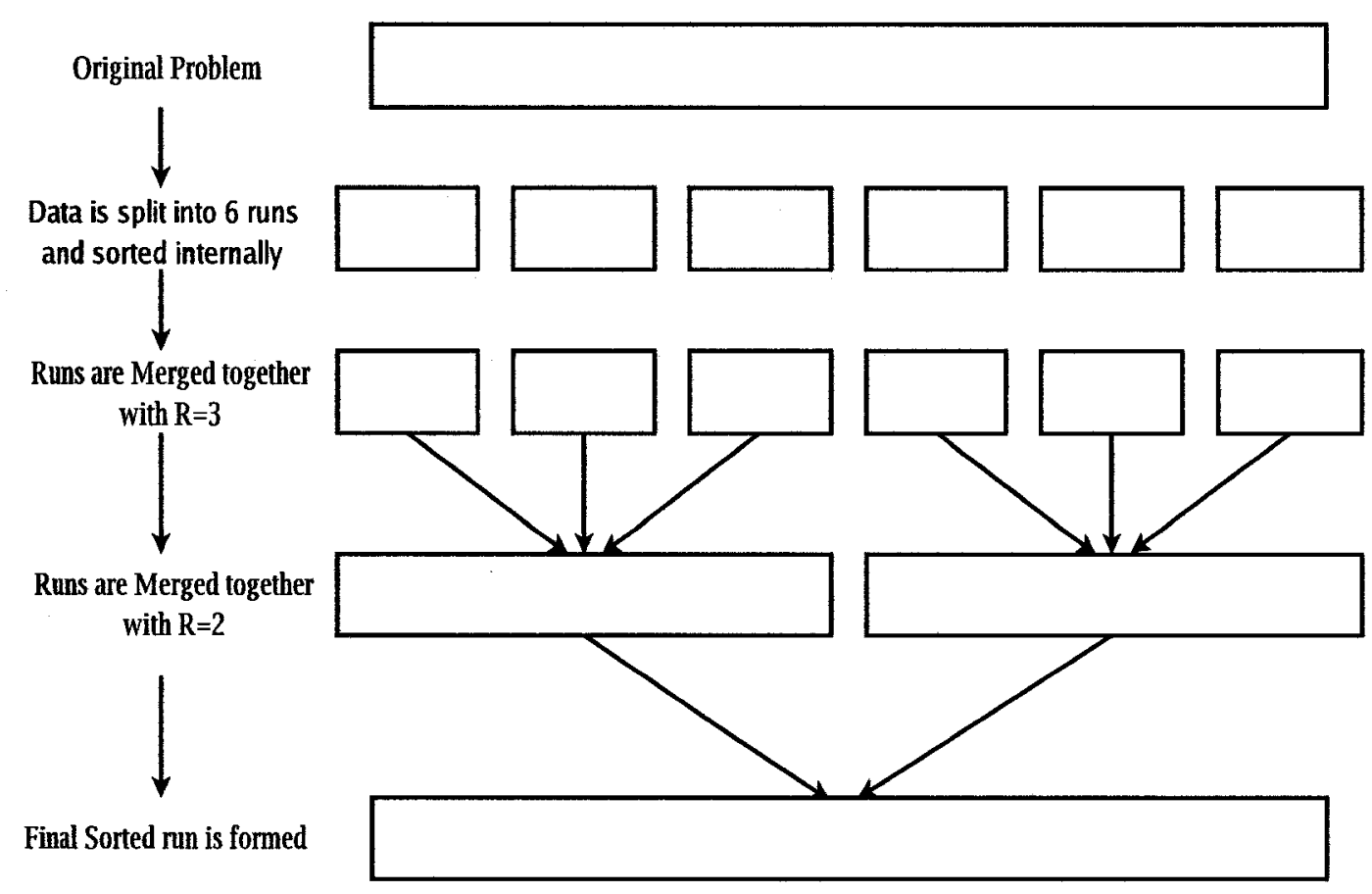

Figure 2.2: Illustration of merge sort with $\frac{N}{M}=6$ and $R=3$ and 2

merge sort. These include the use of multiple disks [37] to increase I/O throughput, "replacement-selection" [33] to form runs of size $2 \mathrm{M}$ and double buffering to overlap $\mathrm{I} / \mathrm{O}$ and computation $[16,15]$. For more details on different merging techniques refer to $[49]$.

Theorem $2.2[1,37]$ The average-case and worst-case number of $1 /$ Os required for sorting $N$ data items using $D$ disks is $\operatorname{Sort}(N)=\Theta\left(\frac{N}{B D} \log _{\frac{M}{B}} \frac{N}{B}\right)$.

\subsubsection{List Ranking}

List-ranking is the problem of finding the distance of each node in a linked list from the last node of the list. It is a basic step on linked lists which occurs in many graph based algorithms. The distance between two nodes can be defined as the number of 
links between these two nodes. Due to irregular memory access, list-ranking has a very poor locality and hence it is difficult to design an efficient parallel or external memory algorithm for list-ranking. There is no obvious way to partition the list into

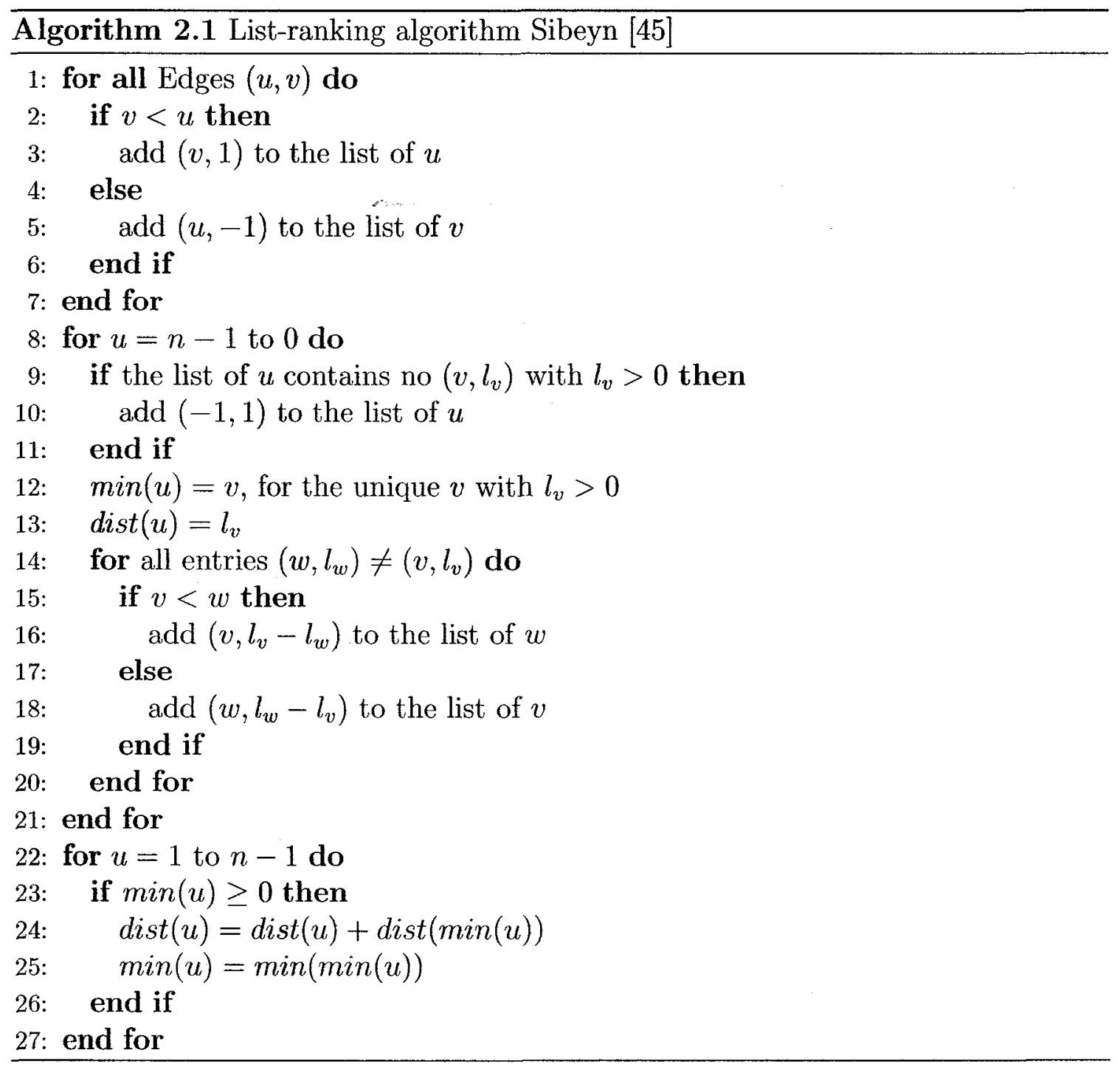

independent contiguous sub lists without knowing the rank of each node. Surprisingly, Most of the external memory algorithms for list ranking are adaptations or simulations of parallel list-ranking algorithms [7, 43]. Sibeyn [45] has developed an algorithm for 
the connected component problem in EM. This algorithm with some modifications can be used for list ranking, viewing the list ranking as a weighted and directed form of connected component. Algorithm 2.1 shows how it works.

Link List:

Input to the Algorithm:

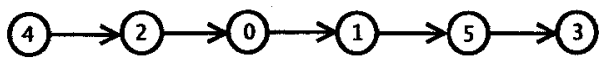

$(4,2),(2,0),(0,1),(1,5),(5,3)$

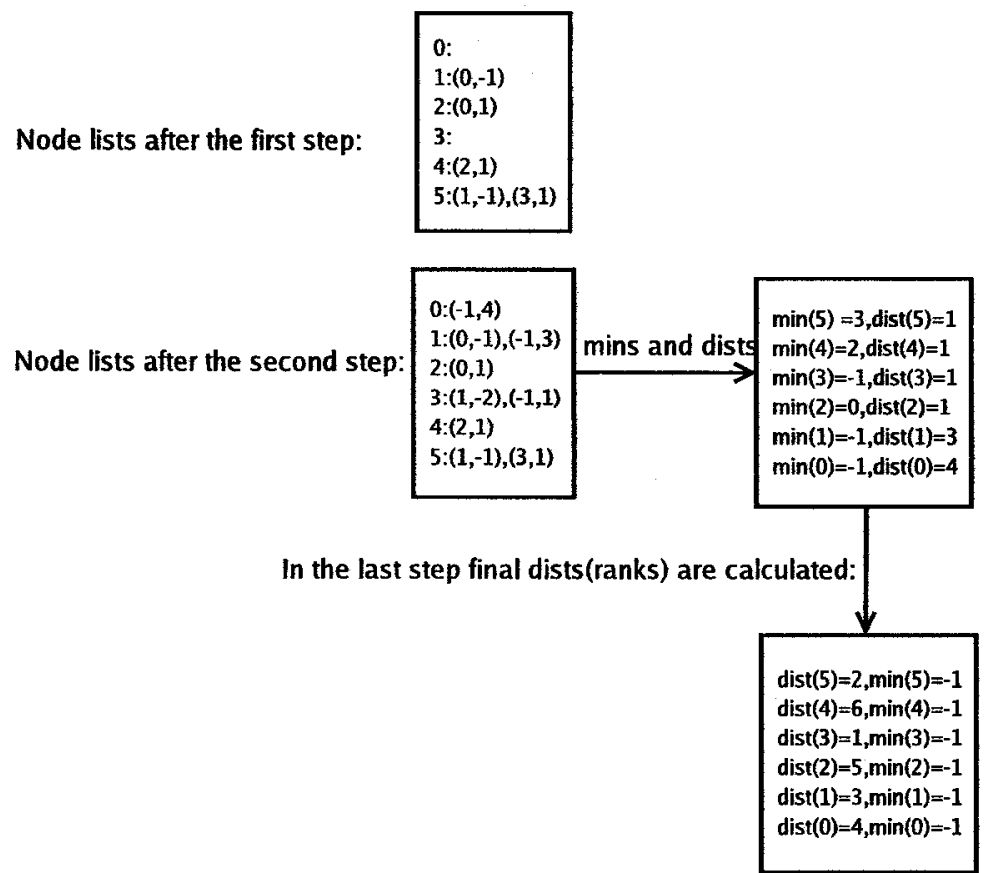

Figure 2.3: An example showing how List Ranking Algorithm 2.1 works

The idea is based on removing nodes (similar to pointer jumping[2]) and keeping track of distances of nodes from each other in separate files. This algorithm consists of three main steps: the first step is the first seven lines of the algorithm, lines 8 to 21 are step two and step three consists of lines 22 to 27 . In the first step node lists are created. Each item in the node lists consists of a node index number and a signed distance value(positive means out going edge and negative means incoming edge). In 
step 2 a dummy node (-1) is added to the end of the list. Note that this algorithm calculates ranks relative to this dummy node. In step 2 each node $u$ is processed and if it has list entries $\left(v, l_{v}\right)$ and $\left(w, l_{w}\right), v<w<u$, then a new entry $\left(v, L_{v}-l_{w}\right)$ is added to the list of node $w$. Step three is the final calculation of distances of nodes from the dummy node. Figure 2.3 demonstrates this algorithm with an example. In [45] it is shown that if $n<\frac{M^{2}}{32 \cdot B}$ then a list of total length $n$ which is presented as a set of edges can be ranked deterministically with $\frac{22 \cdot n}{B}$ I/Os. This algorithm can be implemented using one EM stack and three EM priority queues [38].

\subsection{Implementation Studies}

The work of this thesis extends the results of previous implementation work reported in [30] from a single processor to multiple real processors. Other implementation work in External Memory include LEDA-SM [8], TPIE [48] and STXXL [16] which are I/O workbenches developed to explore external memory algorithm implementations for sequential machines. An experimental work which is closely related to our work is SSCRAP [19] which is a framework for implementing parallel coarse grained algorithms. SSCRAP has been 'extended' [27] to support the simulation of certain parallel algorithms in external memory. We discuss these software projects briefly in the following subsections. 


\subsubsection{TPIE}

TPIE $[4,48]$ was the first real step toward implementation of EM algorithms and data structures in the form of practical libraries and software packages. TPIE provides prototype implementations of many contemporary EM algorithms. Although the original design and claim was to support parallel disks, at the time of writing TPIE does not support multiple processors or multiple disks. TPIE is a modular software with three components: Block Transfer Engine (BTE), Memory Manager (MM) module and Access Method Interface (AMI) module. AMI works on top of $\mathrm{BTE}$ and MM and is responsible for providing the user interface for user programs. TPIE provides basic operations such as scanning, permutation routing, merging, distribution, batch filtering, many (geometric) search data structures such as B+-tree, persistent $\mathrm{B}+$-tree, R-tree, $\mathrm{K}$-D-B-tree, $\mathrm{KD}$-tree, Bkd-tree and matrix operations. The TPIE project is still active.

\subsubsection{LEDA-SM}

The LEDA-SM C++ class library project [8] was aimed at extending the LEDA library to external memory or out of core computation. The goal was to enable the LEDA library to handle large data sets. LEDA-SM consists of a kernel to abstract the secondary memory view (disk I/O), some external memory data structures that are built on top of the kernel and some algorithms which work on these data structures. This library has I/O-efficient implementations of sorting, external memory queue, stack, array heap, radix heap, array, buffer tree, string, suffix array, B+-tree, static graph, matrices and some simple graph algorithms. However, LEDA-SM algorithms and data structures have a very important weakness, they cannot handle more than 


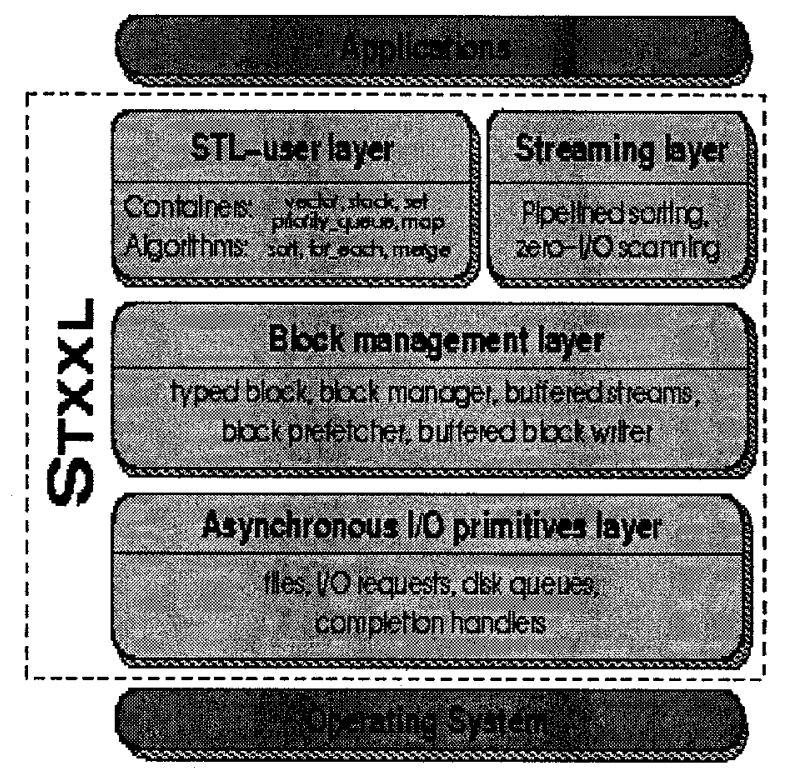

Figure 2.4: STXXL System Software Layers (from [16])

$2^{31}$ data items. LEDA-SM only supports a single disk for its external memory data structures and algorithms. The LEDA-SM project is no longer active [15]. The latest version of LEDA-SM is 1.3 which does not support LEDA releases later than version 4.2 .

\subsubsection{STXXL}

The STXXL $[16,15]$ project aims to provide an external memory algorithms library with high performance and portability. It also aims at addressing some of the weaknesses and lessons learned in TPIE and LEDA-SM development. Some of the features of STXXL are: "transparent support of parallel disks", handling of huge data sizes up to terabyte order, overlapping of computation and $1 / \mathrm{O}$ by using asynchronous I/O. It also has a pipelining capability when multiple external memory algorithms are run one after another. STXXL has a layered design model. The lowest layer is the 
Asynchronous I/O (AIO) primitive layer. This layer abstracts out the details of AIO operation on different operating systems. The next layer is the Block Management (BM) layer which emulates the parallel disk model. The implementation of BM layer is asynchronous and provides the overlapping of computation and $\mathrm{I} / \mathrm{O}$. The third level contains a streaming module and a user interface module. The user interface module provides an interface for external memory algorithms such as sorting, stacks, queues, etc. The streaming module supports pipelining of the external memory algorithms. An interesting feature of the STXXL library is that user access is not limited to the user interface layer. A user can directly use lower layer interfaces as well. For example a user may just make use of the STXXL's AIO layer or BM layer. The performance of STXXL is very attractive judging from our initial tests but it does not itself support multiple processor algorithms at the time of this writing.

\subsubsection{SSCRAP}

SSCRAP [19] is a $\mathrm{C}++$ software environment for implementing parallel coarse grained algorithms based on a BSP-like parallel model called PRO [22]. An extension of SSCRAP to simulate coarse grained algorithms in an external memory setting with reference to the theoretical framework by Dehne et al. $[10,13,12]$ is described in [27]. We will refer to this as SSCRAP-ex. Although based on the framework of Dehne et al., SSCRAP-ex has some limitations and there are some issues which are not clear from $[27]$.

- It is not clear, whether SSCRAP-ex handles communication traffic between virtual processors in an I/O-optimal way.

- SSCRAP-ex cannot run on networks of workstations. Although it can run on 
multiprocessor machines with shared memory, it is not clear whether it can exploit the parallel processing power of these machines.

- SSCRAP-ex has a general limitation in hardware address space. It cannot handle problem instances where problem size is beyond the hardware address space.

In contrast the Parallel External Memory System (PEMS) as described in this work,

- handles the communication traffic in an I/O optimal way.

- runs on networks of workstations and exploits power of real parallel processing.

- has no limitation regarding the problem size except for the total amount of available free disk space to use.

\subsubsection{Previous Simulation Implementation Work}

In $[30,53]$ some experiments are described which tested the practicality of the simulation technique. The result of that project was a software which could simulate a simple parallel program on a single processor machine using external memory. It uses a user implementation of threads to simulate virtual processors. It also uses a set of user built asynchronous I/O functions to handle I/O. POSIX pthreads were used to implement asynchronous I/O. The experiments showed promising, near linear relationship between problem size and simulation time for a sort problem. There are several reasons that we did not use the older code and started over from scratch.

1. That code uses a non-standard, non-portable user implementation of threads.

2. It uses complex user built asynchronous I/O functions. 
3. It does not have the generality and capability of execution in a real multiprocessor environment.

Most of the complexity of the old code is in its threading and asynchronous $\mathrm{I} / \mathrm{O}$ subsystems. Today asynchronous I/O is supported by the Linux kernel and other operating systems, there is also a POSIX interface for doing asynchronous I/O. Today many user space cooperative thread libraries with easy to use interfaces exist. By using these facilities, implementing a system (library) for writing parallel external memory programs is much easier. Now it is possible to focus on issues such as multiple disks and real parallel processing.

\subsection{MPI}

The Message Passing Interface, MPI [20, 34], is a standard API (Application Programming Interface) that can be used to create parallel applications. MPI is designed primarily to support the Single Program Multiple Data (SPMD) model. As MPI is a standard communication library, programs written using MPI are highly portable. MPI has become the leading standard for message-passing libraries. Every vendor of a high performance parallel computer has its own implementation of MPI. There are also a couple of public domain implementations of MPI such as LAM/MPI [6, 46], MPICH $[25,26]$ and Open MPI [21]. LAM/MPI is now in a maintenance state and its development is stopped. Some of the LAM/MPI team members now are working on the Open MPI project. The Open MPI project is supported by many universities as well as commercial companies such as IBM, Sun Microsystem and Cisco Systems. It is aiming at developing a high performance, highly portable implementation of the MPI-2 standard [35]. One of the features of the MPI-2 standard which is important 
to our work is its thread safety and concurrency. This is because in this project we are dealing with a multi-thread multi-processor software that uses MPI as a means of communication between real processors. 


\section{Chapter 3}

\section{BSP Algorithms in External}

\section{Memory}

In this chapter we present the main ideas behind the simulation technique proposed in [12] that transforms a coarse grained parallel algorithm into an efficient parallel EM algorithm which we call EM-BSP algorithm. It optimizes blockwise data access and disk $\mathrm{I} / \mathrm{O}$ and at the same time utilizes multiple processors connected via a communication network or shared memory.

\subsection{Single Processor Simulation Algorithm}

The basic idea of simulation of BSP algorithms in External Memory is the following: assume that the original BSP algorithm runs on $v$ processors, now using some software techniques we create $v$ virtual processors that can run on a real single processor. These virtual processors need to communicate with each other as part of the BSP algorithm. As all of these virtual processors are ruming on the same machine and their message 
sizes can be large, the interprocess communication should be performed through the disk. On the other hand since memory size may not be large enough to hold all of the virtual processors with their data (which is almost always the case), we need to use disk space to swap the virtual processor's context. Algorithm 3.1 shows the main steps used in simulating a simple superstep of a $v$-processor BSP algorithm as a single processor EM-BSP algorithm.

Algorithm 3.1 Single Processor EM-BSP simulation For $j=0$ to $v-1$ do

1. Read the context of virtual processor $j$ into memory.

2. Read packets received by virtual processor $j$ from the disk.

3. Simulate the local computation of virtual processor $j$.

4. Write the packets which are sent by virtual processor $j$ to the disk.

5. Write back the changed context of virtual processor $j$ to the disk.

Theorem 3.1 [12] A $v$ (virtual) processor BSP algorithm $\mathcal{A}^{\prime}$ with $\lambda$ supersteps, local memory size $\mu$, running time $\beta+g \cdot O\left(\frac{N}{v}\right)+\lambda L$, and message size $\Theta\left(\frac{N}{v^{2}}\right)$ can be simulated as a single processor EM-CGM algorithm $\mathcal{A}$ with time $v \beta+O(\lambda v \mu)+G$. $O\left(\lambda \frac{v \mu}{B}\right)$ for $M \geq \mu+B, N=\Omega\left(v^{2} B\right)$, and $v=\Omega(1)$.

\subsection{Multiprocessor Simulation Algorithm}

The simulation techniques described in the previous section has been extended to multiple real processors $[12,30]$. In this case an algorithm which is designed for $v$ processors is simulated on $p$ real processors where $p \leq v$. The result is that each real processor will simulate the execution of $\frac{v}{p}$ processors. Now communication between 
virtual processors is performed through the disk and some other means of communication such as network or shared memory. We also need to swap in the context of a virtual processor as it runs and swap its context out of memory when it is waiting for other virtual processors. Algorithm 3.2 shows the main steps for simulating a superstep of $v$-processor BSP algorithm as a p-processor EM-BSP algorithm. In Chapter 4 we will slightly modify this algorithm to address implementation issues that we encountered (see Algorithm 4.2).

Algorithm 3.2 Multiprocessor EM-BSP simulation For $\mathrm{j}=0$ to $\frac{v}{p}-1$ do in parallel

1. Read the context of virtual processor $i \frac{v}{p}+j$ from the local disk.

2. Read any messages addressed to virtual processor $i \frac{v}{p}+j$ from the local disk.

3. Simulate the computation superstep of virtual processor $i \frac{v}{p}+j$, collecting all generated messages in the local internal memory.

4. Send all generated messages to the required (real) destination processors.

5. Receive all messages addressed to real processor $i$ on behalf of virtual processors $i \frac{v}{p}$ to $(i+1) \frac{v}{p}-1$ in local internal memory and write them to the local disk.

6. Write the changed context for virtual processor $i \frac{v}{p}+j$ back to the local disk.

Theorem 3.2 [12] A $v$ (virtual) processor BSP algorithm $\mathcal{A}^{\prime}$ which communicates via $h$-relations of size $h=O\left(\frac{N}{v}\right)$ with $\lambda$ supersteps/rounds, local memory size $\mu$, computation time $\beta+\lambda L$, communication time $g \alpha+\lambda L$ can be simulated as a $p$ (real) processor EM-BSP algorithm $\mathcal{A}$ with $\frac{v}{p} \lambda$ rounds, computation time $\frac{v}{p}(\beta+O(\lambda \mu))+$ $\frac{v}{p} \lambda L$, communication time $\frac{v}{p} g \alpha+\frac{v}{p} \lambda L$, and I/O time $\frac{v}{p} G \cdot O\left(\lambda \frac{\mu}{B}\right)+\frac{v}{p} \lambda L$ for $M=\Theta(\mu)$, $N=\Omega(v B), B=O\left(\frac{N}{v^{2}}\right), p<v$. 
The parameter $G$ is the ratio between the local computational capacity and the local I/O capacity and the parameter $B$ is the disk block size.

\subsection{Personalized Communication}

One of the requirements of the simulation technique presented in [12] is the message size of $c \frac{N}{v^{2}}$ where $c \geq 1$. This is to ensure that the $\mathrm{I} / \mathrm{O}$ is performed in blocks when reading or writing messages to the disk. On the other hand, it makes it possible to allocate fixed size slots on the disk for messages. But not all algorithms generate all messages with the required message size. To overcome this problem we use the personalized communication or balanced routing algorithm introduced by Bader et. al [5]. The idea is that instead of sending its messages directly to the final destination, each processor breaks the messages into smaller pieces and sends the pieces to different processors with a special order. Then in another round of communication the intermediate processors reorganize the received pieces and forward them to their final destinations.

So each original round of communication will be performed in two rounds of communication. Algorithm 3.3 shows the balanced routing here $m s g_{i j}$ is the message from processor $i$ to processor $j$ and $\left|m s g_{i j}\right| \leq h=\frac{N}{v}$ is the length of the message.

Theorem 3.3 [12] We are given $v$ processors, and $N$ data items. Each processor has exactly $\frac{N}{v}$ data to be redistributed among the processors, and no processor is to be the recipient of more than $h$ data. The redistribution can be accomplished in two communication rounds of balanced communication: (A) Messages in the first round are at least $\frac{N}{v^{2}}-\frac{v-1}{2}$ and at most $\frac{N}{v^{2}}+\frac{v-1}{2}$ in size, and $(B)$ Messages in the second 
Algorithm 3.3 Balanced Routing (from [5])

A. For $i=0$ to $v-1$ do in parallel

1. Processor i allocates $\mathrm{v}$ local bins, one for each processor.

2. For $j=0$ to $v-1$

3. For $l=0$ to $\left|m s g_{i j}\right|$

Processor i allocates the $l^{\text {th }}$ word of $m s g_{i j}$ to local bin $(i+j+l) \bmod v$

4. $\quad$ Processor $i$ sends bin $j$ to processor $j$

B. For $j=0$ to $v-1$ in parallel

1. Processor $j$ reorganizes the messages it received in step A into bins according to each element's final destination

2. Processor $j$ routes the contents of bin $k$ to processor $\mathrm{k}$ for $0 \leq k \leq v-1$

round are at least $\frac{h}{v}-\frac{v-1}{2}$, and most $\frac{h}{v}+\frac{v-1}{2}$ in size.

If $h=\frac{N}{v}$ which is the case for CGM algorithms then the maximum and minimum message sizes for both rounds of communication will be: $\frac{N}{v^{2}}+\frac{v-1}{2}$ items and $\frac{N}{v^{2}}-\frac{v-1}{2}$ items respectively. It should be mentioned that balanced routing is necessary only with certain BSP algorithms that cannot otherwise guarantee balanced messages. 


\section{Chapter 4}

\section{Software Design}

\subsection{Introduction}

The Parallel External Memory System (PEMS) software is based on the simulation of virtual processors as user space threads and direct $\mathrm{I} / \mathrm{O}$ for efficient disk operations. In this chapter we explain our software design and show how different components work and interact together in PEMS. The input to our system is an MPI program implementing a BSP algorithm. In such a program, MPI is responsible for interprocess communications. Most of the MPI functions are for sending and receiving messages. To run this program under PEMS, we replace each MPI call by a corresponding External Memory subroutine call. These EM calls, in turn and when needed, incorporate MPI calls. The communication is managed between processes using the disk, memory buffers and communication network; this is the main challenge in the design and implementation of PEMS. Figure 4.1 shows a simple MPI program and Figure 4.2 is the same program converted to a PEMS program.

In this initial version of PEMS, our focus has been on confirming the high level 
Figure 4.1: A simple MPI program

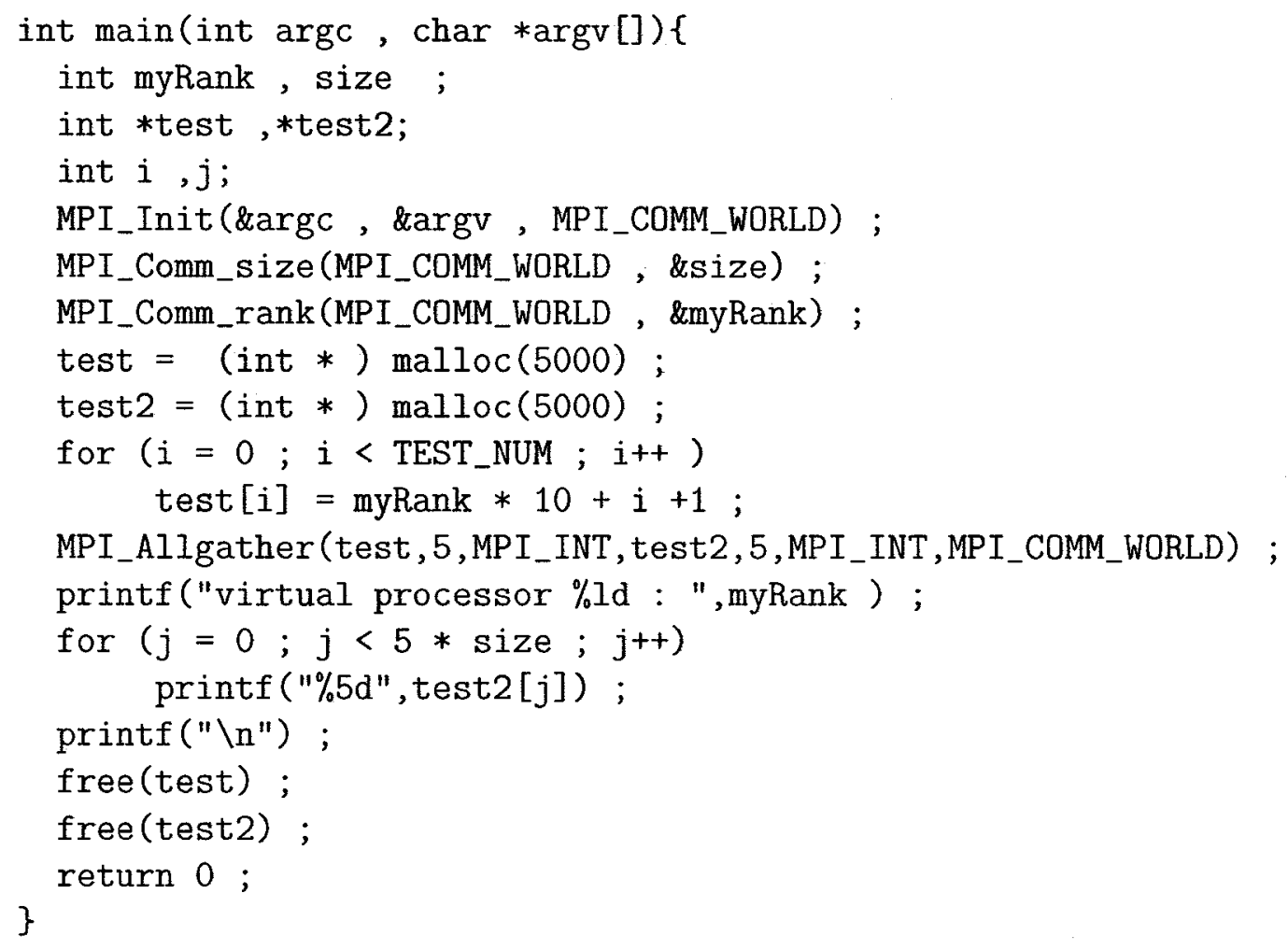

behavior of the simulation approach, that is to confirm that the behavior of PEMS scales predictably as problem sizes and the number of real processors vary. This involves both running time and disk space usage behaviors. We have not been overly concerned about optimization in this initial version of PEMS. In Chapter 6 we discuss possible optimization areas.

Figure 4.3 shows the layered software design of PEMS. The user program is shown as the top layer in the diagram. Layer 2 incorporates the external memory communication primitives. It is assumed that all MPI calls in the original user program are replaced by a call to a similar PEMS function in this layer. Layer 3 has four major features. The Disk I/O Subsystem is responsible for reading and writing data blocks 
Figure 4.2: MPI program in Figure 4.1 converted to PEMS program

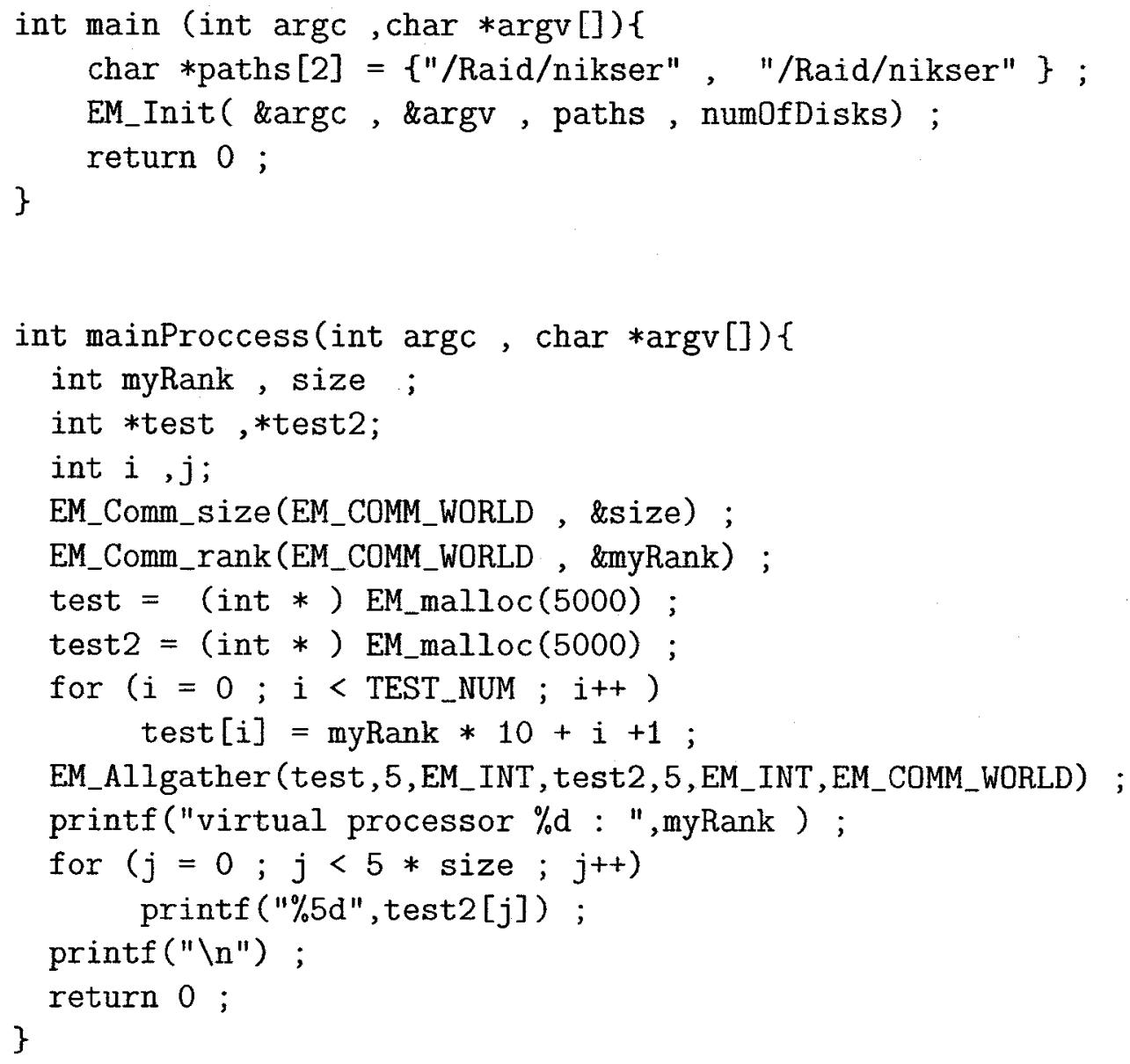

to disk efficiently. The Memory Management Subsystem is responsible for allocating memory for virtual processor data. It is also responsible for swapping out and swapping in this data when requested. The Process Management Subsystem is responsible for creating, scheduling and synchronizing virtual processors. The Open MPI library [21] is used for communication between real processors and also for starting the software on multiple machines. In subsequent subsections we will discuss layers 2 and 3 in more detail. 


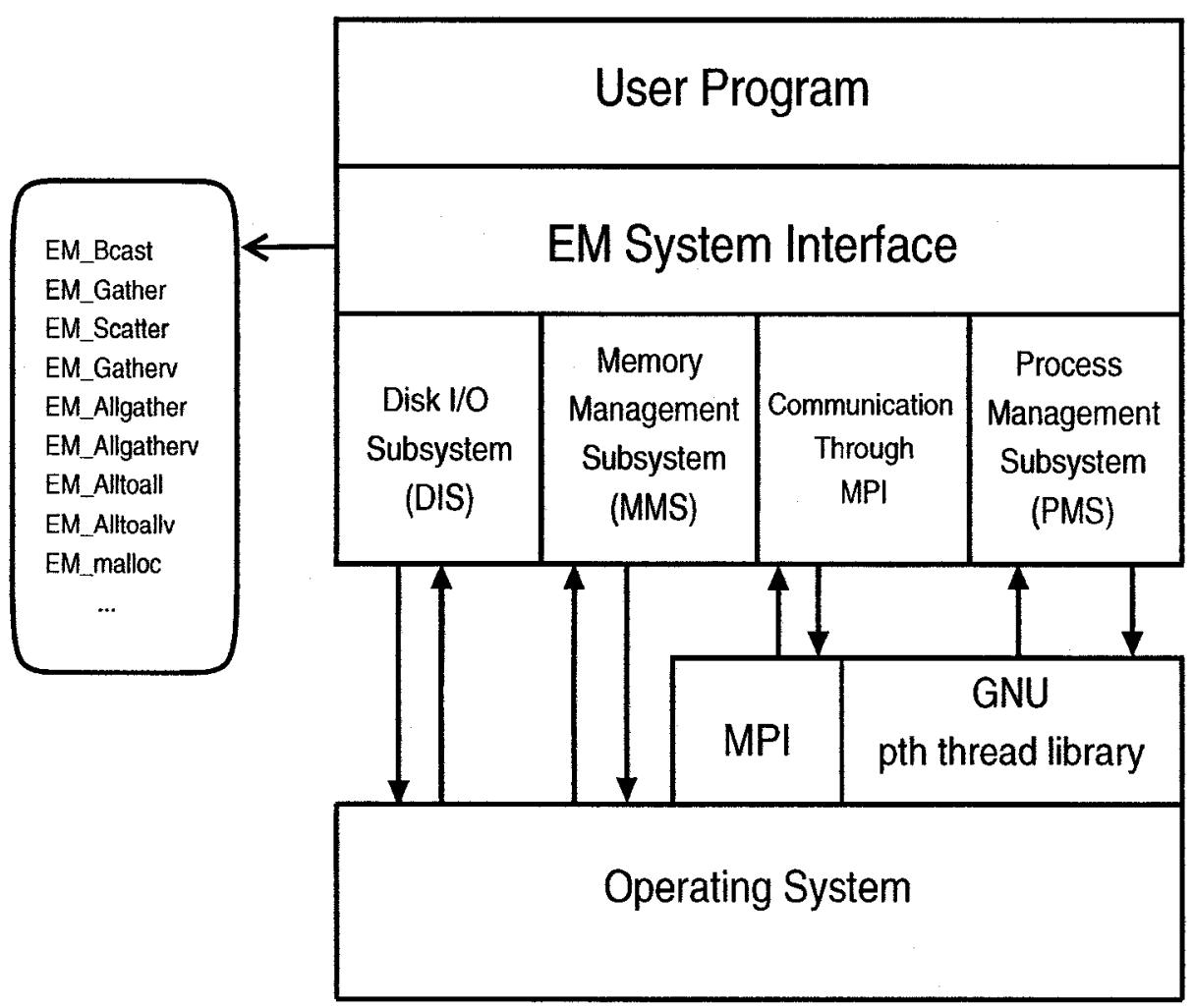

Figure 4.3: PEMS System Software Layers

\subsubsection{Practical Considerations}

Before we provide the details of software design and implementation, we want to mention some deviations from the theoretical simulation algorithms because of practical considerations. The first thing is that in MPI we must have a buffer of appropriate size in order to send or receive a message. Secondly, in practice no virtual processor can send or receive messages larger than its local memory. This is as a result of the previous constraint. So the parameter $h$ in the h-relation in BSP computations is $h=\mu_{d}$ where $\mu_{d}$ is the virtual processor data memory. Next we require some minor changes in the simulation algorithms. Algorithm 4.1 shows the main steps for simulating a superstep of a $v$-processor BSP algorithm as a single processor EM-BSP 
algorithm that we use in PEMS.

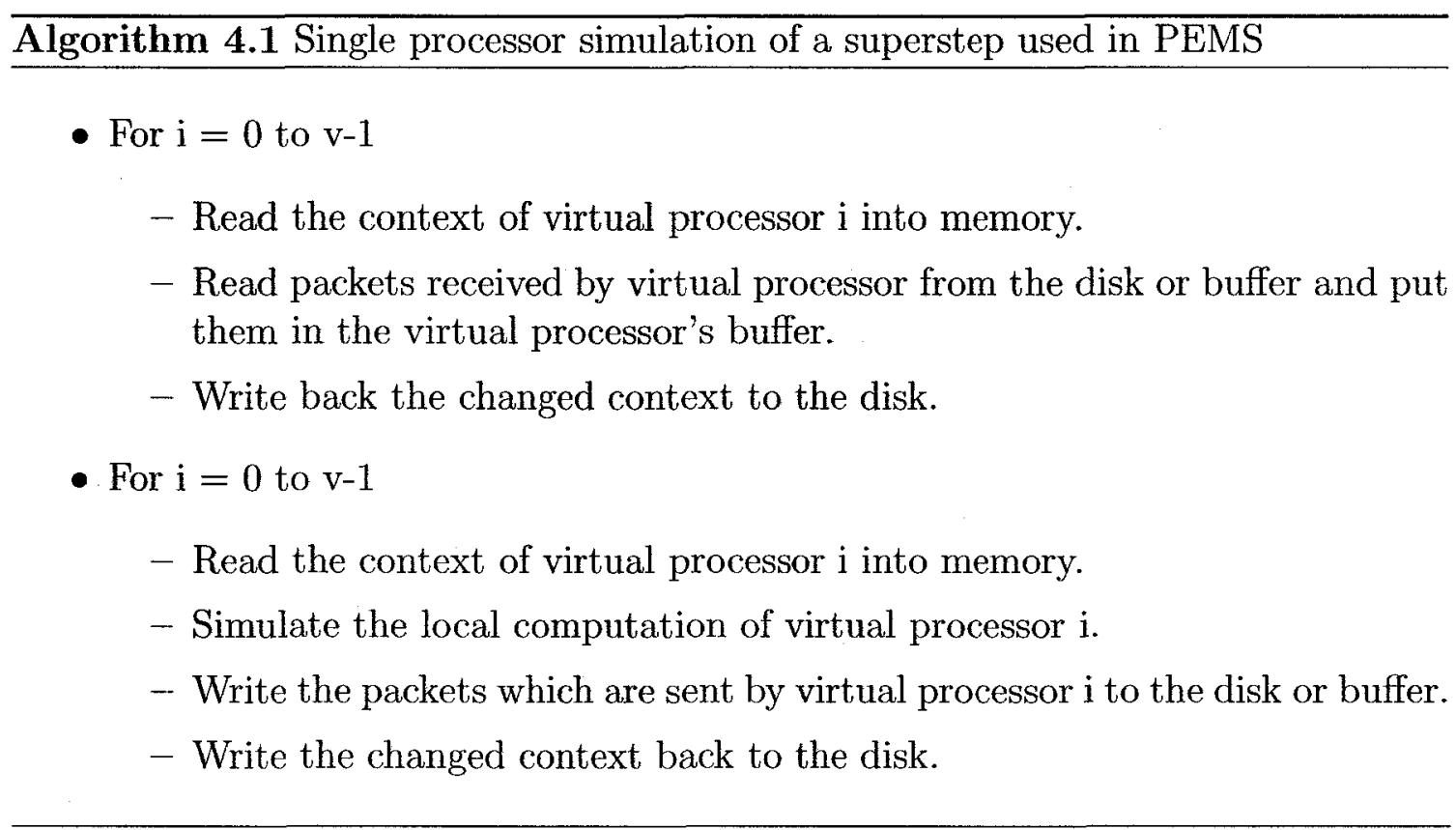

There are two differences between Algorithm 4.1 and Algorithm 3.1 in Section 3.1. 1) We use a memory buffer instead of disk for interprocess communication whenever the messages are small enough to keep them in the memory buffer, and avoid disk I/O for small messages. This shared buffer is accessible by all virtual processors in a machine. 2) We read and write the context of each virtual processor twice in each superstep. This is because of the need for synchronization between virtual processors. Each virtual processor should wait until all virtual processors have read the previous superstep messages from the disk before they start writing new messages, otherwise they may overwrite a message which has not yet been received by its destination virtual processor. This of course can also be solved by using twice the needed disk space: half of the space is used for the current superstep messages and the other half is used for the next superstep messages. We chose to use an additional round 


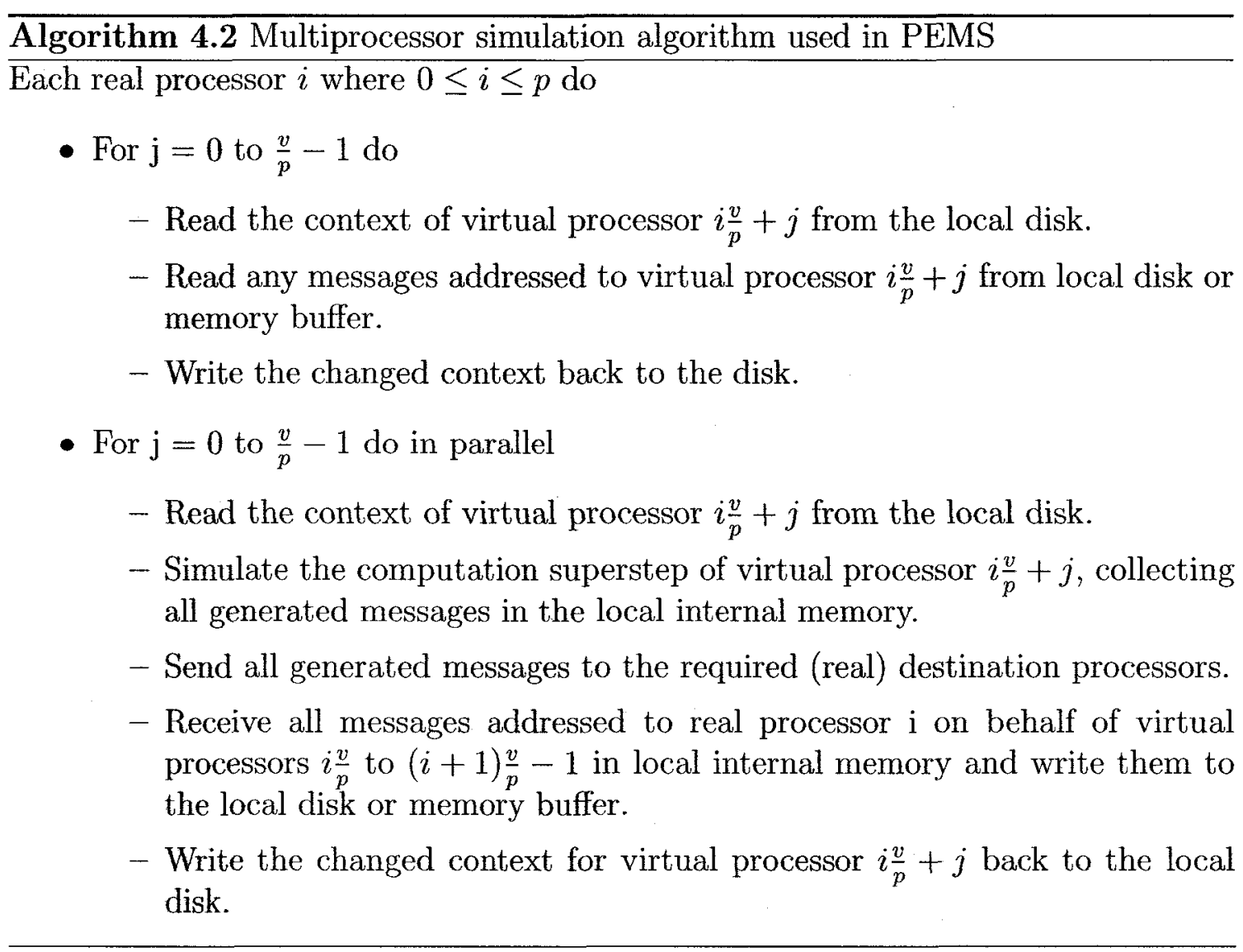

of communication to avoid doubling the disk usage. In practice not all MPI-like EM communication primitives need the two rounds of $\mathrm{I} / \mathrm{O}$. We use another technique to reduce $\mathrm{I} / \mathrm{O}$ activities in some $\mathrm{EM}$ communication primitives which is described in Section 4.5.3.

Algorithm 4.2 shows the main steps for simulating a superstep of a $v$-processor BSP algorithm as a p-processor EM-BSP algorithm which is used in PEMS. This algorithm differs from Algorithm 3.2 in the same way as in the single processor case, shared memory buffer and a second round of context swapping is added. 


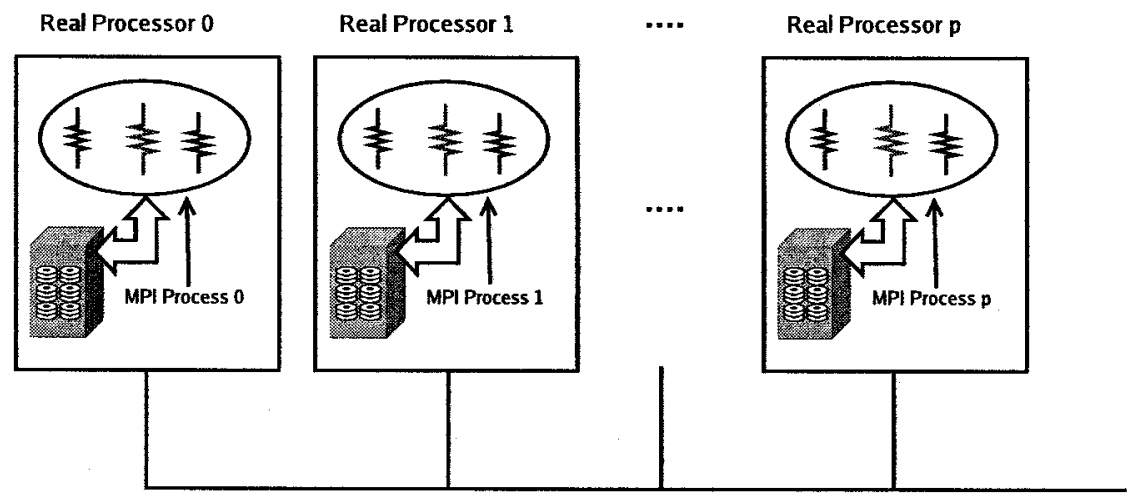

Figure 4.4: PEMS on $p$ real processors and 3 threads per processor.

\subsection{Virtual processor simulation and process man- agement}

The PEMS system can orchestrate a simulation on one or multiple real processors. The GNU pth thread library $[18,23]$ is used on each real processor for simulating virtual processors as user space threads. Each virtual processor is represented by a user level thread, and that thread runs a copy of the modified MPI-based user program. Figure 4.4 illustrates PEMS system running on $p$ real processors with three threads per processor. Modifications to the user program include replacement of MPI calls with the corresponding PEMS calls and usage of the dynamic memory provided by the PEMS Memory Management Subsystem for data sets, buffers and workspaces. The PEMS Process Management Subsystem consists of a set of functions to initialize, start, synchronize, manage, and schedule threads. These functions are implemented using the GNU pth thread library which provides service for the creation and cooperative scheduling of threads. Scheduling of threads requires the use of the memory management subsystem - when a thread stops running, its data must be 
swapped out and the data for the new thread must be swapped in.

Starting, stopping and coordination of different processes on different machines is achieved using the open MPI library [21]. Each virtual processor has a global identification number which we will call its PEMS rank, as well as a local thread number within its user space process, and an MPI rank that identifies which MPI process contains it. The PEMS rank of a virtual processor is computed based on the MPI rank of the process and its local thread number. When virtual processors are communicating, their PEMS ranks are used to distinguish them from each other. It is possible to change the total number of virtual processors by changing the number of threads in each MPI process or by changing the number of real processors. Number of real processors is an input to the MPI system and number of virtual processors (threads) per real processor is an input to the PEMS system.

\subsection{Context Switching and Memory Management}

As a multi-threaded program runs, context switching between threads occurs whenever a thread stops executing and another thread starts execution. From the kernel point of view all of our threads on a single real processor are within one process and context switching is done by the GNU pth-thread library, which is part of the running program in the user space. We have assumed that each virtual processor (thread) needs as much physical memory as it can allocate and hence we face two problems: (1) If each virtual processor allocates as much physical memory as it can, there will not be enough physical memory for all virtual processors, and the operating system will start allocating memory from its swap space or virtual memory. Then each time a thread starts execution, the operating system will swap in the needed pages of data 
from the disk swap area. This is done based on a page fault mechanism which may not be efficient for our purposes. We need an EM-efficient swap out of the previous thread's data and swap in of the new thread's data. (2) Even if the operating system was able to perform swapping efficiently, the total amount of memory needed for all virtual processors could be well beyond the virtual address space of the combined operating system and the hardware. This is specially true for 32-bit machines. Therefore, we need complete control over PEMS' physical memory and all of the disk activities related to the execution of the PEMS program.

To address these issues, we have implemented a Memory Management System which carfully allocates physical memory at the beginning of the program execution (initialization phase). Half of the available memory is used as a buffer for communication purposes and for assembling and disassembling messages (see Section 4.5.2). The other half of the available memory is reserved for use by the virtual processors that are currently running. A virtual processor (or the copy of the user program that it is simulating) can allocate memory from this area by calling EM_malloc(). In fact each virtual processor does not necessarily need to allocate all of its memory requirements from PEMS. Instead it can only allocate those variables from PEMS which should be preserved between supersteps. If there is still free memory available in the system and a virtual processor needs some temporary data structures in a computation superstep, it can allocate memory from operating system and frees this memory before calling any EM communication primitive. Comparing to the case where all of the memory needs of virtual processors is allocated from PEMS, this technique reduces the number of I/Os required for swapping.

At the end of each superstep EM_yield() is called by each virtual processor. This 
Process Management Subsystem function first takes care of swapping the data and then it calls on the pth library to do the real context switching.

\subsection{Disk I/O Subsystem}

This layer is created in order to make the higher layers of the system independent of specific operating system I/O calls. This makes the system more flexible and portable. It gives us the ability to use third party I/O libraries without making any changes in the higher layers of the software. During our studies we have implemented different versions of disk $\mathrm{I} / \mathrm{O}$ functions. We have implemented direct $\mathrm{I} / \mathrm{O}$ based on the native Linux direct $\mathrm{I} / \mathrm{O}$ which is supported in kernel versions 2.6 and above. We also experimented with Linux asynchronous I/O and synchronous I/O. Based on our experimental studies we decided to use direct synchronous I/O for swapping and buffered synchronous I/O for messaging. Asynchronous I/O makes the system more efficient when used with multiple disks. Note that use of asynchronous I/O requires careful synchronization between virtual processors so that a virtual processor does not read data before another virtual processor finishes writing to the disk.

\subsection{PEMS System Interface and Messaging}

This layer is the most important layer in the implementation. While the contexts (local data) of each virtual processor can be swapped between RAM and disk using efficient streaming $\mathrm{I} / \mathrm{O}$, this is not genereally true for delivering messages between virtual processors in the simulation. In this layer we have implemented external 
memory equivalents of the MPI communication primitives and functions. In particular we have implemented most of the collective communications such as EM_Bcast, EM_Gather, EM_Scatter, EM_Alltoall, EM_Alltoallv. All of the PEMS functions have the same number and type of parameters as their MPI counterparts. This makes it easier to transform an MPI program into a PEMS program. In fact we believe that there are simple automated ways that can help programmer to do this easier. For example a pearl script which parses the MPI program and replaces all MPI prefixes with EM prefixes. We have also implemented EM data types corresponding to the MPI data types (e.g. MPI_INT, MPI_CHAR). As we mentioned earlier communication is done through the disk, memory buffer, network, or a combination of them depending on the communication function. If the two communicating virtual processors are on the same machine then they communicate through the disk or memory buffer. If they are on two different machines they communicate through the network and disk or memory buffer. As discussed in Section 4.3 we split the available physical memory into two partitions. One partition is used by the virtual processors and the other partition is used by the PEMS as a large buffer. Any message which fits into this buffer is kept there instead of being written to the disk. Since no virtual processor can receive a message bigger than its data memory size and the buffer size is equal to the virtual processors' data memory size, it is possible to keep most of the messages in this buffer. The exceptions are messages generated by the functions EM_Alltoall and EM_Alltoallv. In this type of communication, potentially all of the virtual processors are generating messages which are destined to all other virtual processors and each virtual processor may receive different number of messages with arbitrary sizes. So each of the $\frac{v}{p}$ virtual processors running on a real processor may receive a message 
as large as the shared memory $\left(\mu_{d}\right)$ this message is different from the messages that other virtual processors on the same machine receive. Since it is not possible to keep all of these messages in the shared memory buffer, they are written to the disk. The destination virtual processor will read the relevant messages from the disk when it becomes active. This requires maintaining information pertaining to where these messages are stored on the disks, as well as efficient retrieval mechanisms of the disk blocks corresponding to these messages. These details are discussed in Subsection 4.5 .2 .

As mentioned before, communication between two virtual processors which are running on two different machines is done through the network and buffers (either disk or memory). Here is how it works: the sending virtual processor generates the message and calls the relevant PEMS function for communication. PEMS determines the MPI rank of the destination virtual processor and sends the message to the destination real processor. But as the final destination virtual process may not be running at the time when messages arrive, the current running virtual process at the destination machine receives the messages on behalf of the destination virtual process and saves the messages in the memory buffer or on the disk. The actual receiver can read the messages later whenever it becomes active. Now we explain how communication primitives operate in more detail using two of the primitives as examples.

\subsubsection{EM_Allgather}

As the first example we describe how EM_Allgather works. The complete syntax for this primitive is the following: 
int EM_Allgather(void *sendBuff,int sendCount,EM_Datatype sendType,void *recvBuff,int recvCount,EM_Datatype recvType,EM_Comm comm).

This function performs in an analogous way to the corresponding function Allgather() of the MPI standard [35]. In this case instead of the original real processors communicating over a network using MPI, we have virtual processors communicating. These virtual processors may be running on one or more real machines and their aggregate memory usage may be many times larger than the real memory in the system. Each virtual processor sends "sendCount" items of the type indicated by "sendType" to all other virtual processors and each virtual processor receives "recvCount" items of type "recvType" from all virtual processors. The total number of items received by each virtual processor will be $v \times r e c v$ Count. The total size of this message is less than or equal the local memory size of a virtual processor. PEMS handles this communication as follows:

1. Active virtual processors communicate through an MPI_Allgather to identify the virtual processors which are active and participating in the current round of communication (there is only one active virtual processor in each machine at any time).

2. Active virtual processors send their messages to each other through another MPI_Allgather. The received messages are copied to the shared buffer in a proper offset based on the sender's rank.

3. The active virtual processors reach a barrier, yield to other virtual processors inside their real processor and wait until all virtual processors perform these three steps. 
4. After all virtual processors reach the barrier, they become active and the received messages are copied to the user buffer from the shared buffer. Then they reach another barrier to let other virtual processors receive the data from the global memory buffer.

5. When all of the virtual processors reach the second barrier the EM_Allgather is complete.

If simulation is performed only on one machine (single processor simulation) no MPI communication is needed. Each virtual processor simply writes its message to the shared buffer, yields to other virtual processors and waits at a barrier. Then virtual processors become active again one by one and copy the entire messages sent by all virtual processors from the shared buffer to the user buffer and again yield and wait. After this second barrier the control returns to the user program.

\subsubsection{EM_Alltoallv}

The most complicated communication primitive is EM_Alltoallv. This primitive allows all virtual processors to send messages to all other virtual processors. The messages to different processors can be of different sizes. The only restriction is that they need to fit into the local buffers of each virtual processor (i.e., they need to obey the hrelation requirements of the underlying $\mathrm{BSP}$ model). As real processor $p_{i}$ simulates $\frac{v}{p}$ virtual processors, in each round of communication it will receive all messages addressed to the virtual processors which it simulates. It is possible, in our current implementation, that a user thread representing virtual processor $v_{i j}$, associated with the real processor $p_{i}$, is called on to handle the messages addressed to all of the virtual processors in $p_{i}$. In that case the total size of the messages received by $v_{i j}$, on behalf 


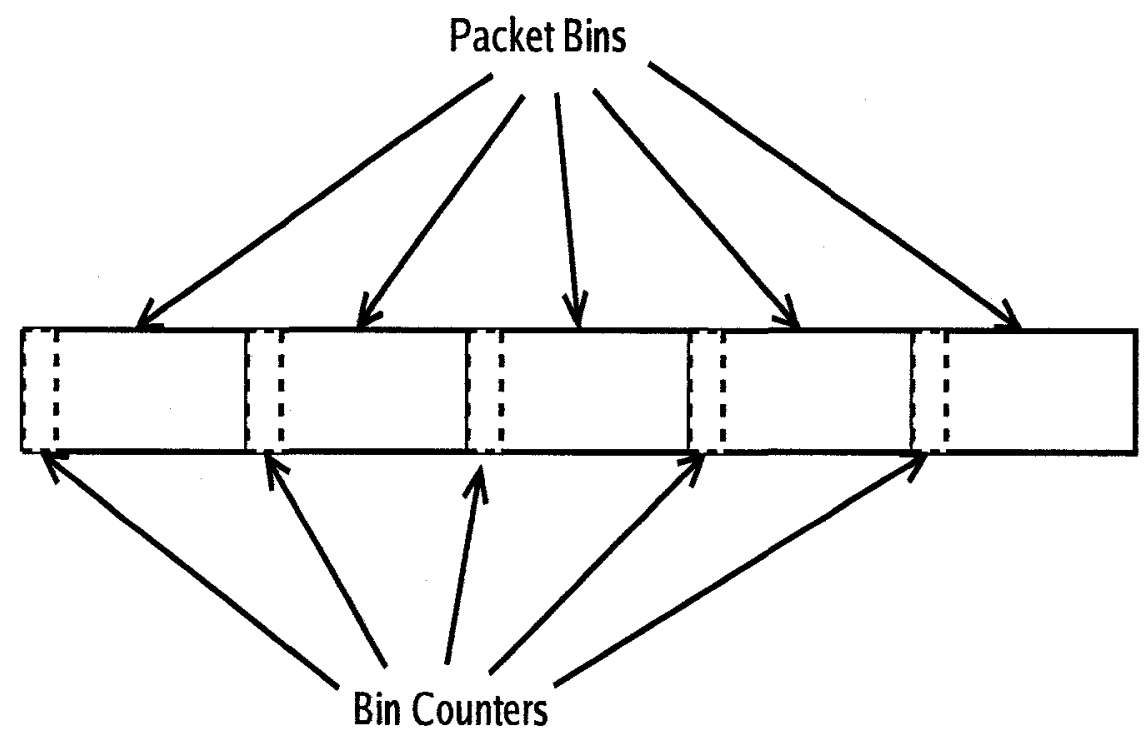

Figure 4.5: Balanced routing bins and bin counters in Alltoallv

of all the virtual processors in $p_{i}$, may be more than the total physical memory of $p_{i}$. More precisely, let the data memory of each virtual processor be $\mu_{d}$, then the maximum message size is bounded by $\mu_{d}$. There are $\frac{v}{p}$ virtual processors in each real processor $p_{i}$, so $p_{i}$ may receive a total of $\min \left(p, \frac{v}{p}\right) \times \mu_{d}$ messages which may be beyond the real processor's RAM capacity. If we use the personalized communication algorithm by Bader et.al. [5] (see Section 3.3) then the message size for each virtual processor is bounded by $\frac{\mu_{d}}{v}+\frac{v-1}{2}$. Then the maximum message size for each real processor is $O\left(\min \left(p, \frac{v}{p}\right) \times \frac{\mu_{d}}{v}\right)$ which is $O\left(\mu_{d}\right)$. This is within reasonable limits and can be handled by a real processor. During the personalized communication the memory buffer is used as a working area for assembling and disassembling messages and packets. Now we describe how EM_Alltoallv handles the communication in detail:

1. Each active virtual processor partitions the shared buffer into $v$ bins. At the 
beginning of each bin, an integer is allocated to be used as a counter. The counters are initialized to zero. Each counter always indicates the current number of packets in the bin. Figure 4.5 illustrates this partitioning with $v=5$.

2. The sent message (in the user buffer) is split into packets and packets are distributed among the bins according to the balanced routing algorithm (see Section 3.3). Each packet contains the source and destination's rank, sequence number and the size of data in the packet payload. The maximum packet data size or PAY_LOAD_SIZE is a compile time parameter of the system. The current PAY_LOAD_SIZE is 128 bytes. We tested PAY_LOAD_SIZE of 256 bytes and observed very small improvement in efficiency. But by increasing the size of packets we also increase the possibility of underfull packets during the communication. This also can cause problems fitting packets in the bins by wasting the available space in the bins.

3. The data area of the virtual processor is swapped out. This area will be used for assembling and disassembling messages.

4. Active virtual processors communicate through an MPI_Allgather to identify the virtual processors which are active and participating in the current round of communication (there is only one active virtual processor in each machine at any time).

5. Using MPI_Alltoall(), active virtual processors route each of their assembled bins to an intermediate real processor destination.

6. Each active virtual processor, as an intermediate receiver, receives the bins on behalf of all virtual processors running on its real processor and writes the bins 
to the disk in a specific offset based on the sender's and the receiver's rank.

7. Each active virtual processor yields to other virtual processors and waits at a barrier (barrier synchronization) until all the threads (virtual processors) in the real processor communicate their initial bins.

8. After all virtual processors reach the barrier, they become active again, read the bins from the disk and reorder the packets in new bins based on the final destination of the packets.

9. Active virtual processors communicate to identify the participating virtual processors in the current round of communication.

10. They route the bins to the final real processors via MPI_Alltoall.

11. Each active virtual processor receives all messages destined to its real processor on behalf of all virtual processors in the real processor. The messages are written to the disk based on the final receiver's rank.

12. Virtual processors synchronize through another barrier.

13. After barrier each virtual processor becomes active, swaps in the user data area, reads the received bins from the disk and unloads data from the packets and copies them to the user buffers.

14. User's data area is swapped out again and virtual processors go through another barrier synchronization.

15. After the last barrier EM_Alltoallv is complete and control is returned to the users program. 


\subsubsection{Direct delivery to context}

Direct delivery to context on the disk is a technique that we have used whenever possible to reduce I/O. This means that while the user's data area of a specific virtual processor is swapped out of the memory and is on the disk, the virtual processor's thread becomes active, reads the received messages from disk or shared buffer and copies them directly to the user's data area on the disk. This requires the computation of the user's buffers addresses on the disk. This is possible because user's buffers are allocated using EM_malloc from the PEMS memory management system. We can keep track of user's buffers on the disk and in the memory. This technique can save one round of swapping out or one round of both swapping in and swapping out for each virtual processor.

\subsection{Discussion on Design Choices}

In this section we will highlight some of the design alternatives for different components of the PEMS and provide some reasoning behind our choices. We also shed light on some possible guidelines for enhancing PEMS in the future based on our current experience. This section consists of several subsections each of them discusses one of the influential issues in the design of PEMS. We begin our discussion with one very basic choice that we had when we started the project.

\subsubsection{Representation of virtual processors}

At the very top level, we had two choices for our software design:

1. simulate each virtual processor as a separate thread, using this method each 
virtual processor has a clear identity and context in the system.

2. view a virtual processor as a function which executes in each superstep. In this method each virtual processor is a function and the programmer must take care of its identity and context as a virtual processor.

We decided that managing the data and context of an independent virtual processor as a thread is easier than managing it as a function. Using tools and services in the threading library, it is easier to give virtual processors an identity. Moreover, the complex task of switching and synchronization between virtual processors is also handled by the thread library.

Another important possibility with threads is concurrent processing and overlapping of $\mathrm{I} / \mathrm{O}$ and computation. We did not explored this aspect of threading in the current version of PEMS and this remains as a possible future expansion of the system.

\subsubsection{Choices for Communication}

There are two types of communication in the system:

Internal communication: Communication between virtual processors which are within a real processor.

External communication: Communication between virtual processors which are running on different real processors.

There are several options on how to perform communication in PEMS:

Option 1: We assign the job of communication (either internal or external) to a separate process (a kernel level thread). As virtual processors generate data for 
communication, they send them to the communication process and that in turn decides if those data should be communicated internally or externally. Here we also need an interprocess communication mechanism to send data from the simulating process to the communication process.

Option 2: As the internal communication is fairly simple, at the end of each superstep each virtual processor can submit its internal communication to the $\mathrm{I} / \mathrm{O}$ subsystem and send its external communication to the communication process. In this approach the communication process is responsible only for external communication. But now the problem is that the communication management should be performed in two different processes and this increases the complexity of the system.

Option 3: Internal communication is done as in Option 2 but the external communication is done by the main thread of the process which simulates virtual processors. This avoids the need for interprocess communication. In this approach the main thread also has a synchronizing role. It should wait until communication data from all virtual processors are written to the disk and it also should make sure that all external communication data are received by peer processors and written to the disk or the memory buffer, before the next superstep starts. The problem with this approach is that all the data, even those messages whose destinations are another machine, should be written to the disk or shared buffer before the main thread can send them to their destination. This may reduce the efficiency of the system and increase unwanted disk I/O or memory copying. 
Option 4: All the internal and external communication is done by the current running threads on all machines. We chose this approach for communication between virtual processors. Each running virtual processor calls the communication routines as subroutines. Communication routines classify messages, communicate with other running virtual processors and gather all the messages sent to their real processor (on behalf of all virtual processors being simulated in this real processor). This approach has the following advantages: First, messages to other machines are sent out as they are generated so there is no additional disk $\mathrm{I} / \mathrm{O}$ or buffer activity for them. Second, there is no need for a separate process to do the communication. Third, each time virtual processors communicate through MPI, they can also go through a synchronization phase with other virtual processors and in fact all real processors can be synchronized without the need to wait for the main threads of the processors.

\subsubsection{A Discussion on Asynchronous/Direct Network Communication}

There is a possibility of using a direct asynchronous blockwise communication over the network. By direct we mean a communication scheme which does not copy data from user buffers to system buffers, but instead directly sends data from the user buffers over the network. The asynchronous scheme may alleviate the need for balanced routing which can eventually reduce amount of disk I/O, but there are some challenges and problems as follows:

1. If we want to use collective MPI primitives, we have to stick with the current synchronous scheme. Otherwise we have to implement a library of non-blocking communication over the network since there is no asynchronous or non-blocking 
collective primitive in MPI.

2. Avoiding kernel buffering in the kernel network stack implies that there should be such services in the kernel as there is for direct disk $\mathrm{I} / \mathrm{O}$. We are not aware of such a service at least in the current Linux kernel.

3. In the BSP model of computation processors communicate at the end of each superstep via h-relation and in practice the messages are generated and ready to be sent suddenly at the end of superstep, not gradually. Virtual processors do not have any computation to do at that time even if the communication is non-blocking. So in order to overlap communication and computation the scheduling of virtual processors should also be changed.

4. Usually when messages are broken into smaller pieces, there is more overhead involved. So we are not sure if it is worth adding this overhead to the system.

5. If there is not enough room in the memory to receive a multi-block message and we receive them one block at a time, then we have to write the message to disk in several parts which increases seek times and overall I/O times.

6. In PEMS the real bottleneck is the disk $\mathrm{I} / \mathrm{O}$, so we think the focus should be on optimizing disk I/O.

\subsubsection{Direct and Asynchronous I/O}

Direct I/O allows the results of an I/O to be written to the user space directly without first being buffered and then copied by the operating system. This is clearly desirable from a speed and overhead point of view. However, incorporating Linux direct I/O 
imposes constraints on the buffer size, alignment and also on the file offset especially when we want to use disk I/O functions such as pread or pwrite. The buffer size must be a multiple of the disk block size and it must also be aligned on a memory address which is a multiple of the disk block size. The same constraint also applies to the offset of the file that we want to write to or read from. This makes implementation of the PEMS versions of certain MPI-like collective primitives very difficult and inefficient, as we often need to write or read to/from a file at arbitrary offsets. We also need to be able to read and write at arbitrary addresses in the buffer space. In order to overcome these difficulties we decided to use two types of disk I/Os, direct I/O for swapping the context data and buffered $\mathrm{I} / \mathrm{O}$ for communication purposes.

Computation and I/O can be overlapped by using asynchronous I/O. But in EMBSP computation, I/O activities happen at the end of each superstep. It means, when a virtual processor reaches the superstep boundary it submits its $\mathrm{I} / \mathrm{O}$ and then it should wait for other virtual processors. So even if the $\mathrm{I} / \mathrm{O}$ is done through nonblocking calls, there is no computation that the current virtual processor can perform. As in the current version of PEMS there is only one virtual processor in the machine's memory at a time, the overlapping of computation and I/O cannot be achieved. To overcome this problem, PEMS should have at least two active virtual processors in the memory. This requires a change in the swapping and scheduling routines of the system and can be an area of future work.

PEMS contains implementations of the four disk I/O combinations: direct synchronous, direct asynchronous, buffered synchronous and buffered asynchronous I/O. These have been implemented using operating system facilities and the pth_pread /pth_pwrite functions of thread library, which prevents the process from being blocked 
Table 4.1: Advantages and disadvantages of kernel and user level threads

\begin{tabular}{|l|l|}
\hline Kernel Level Threads & User Level Threads \\
\hline $\begin{array}{l}\text { Advantage } \\
\text {-Possibility to harvest the power of } \\
\text { multiprocessor machines. }\end{array}$ & $\begin{array}{l}\text { Advantage } \\
\text {-Cooperative scheduling by programmer } \\
\text { which makes thread management and } \\
\text { synchronization much easier. }\end{array}$ \\
& $\begin{array}{l}\text {-Easier coding and debugging. } \\
\text {-Faster switching between threads. }\end{array}$ \\
\hline $\begin{array}{l}\text { Disadvantage } \\
\text {-Preemptive Scheduling by kernel leads } \\
\text { to harder management and } \\
\text { synchronization. } \\
\text {-Harder to develop the code and } \\
\text { debug. }\end{array}$ & $\begin{array}{l}\text { Disadvantage } \\
\text {-More overload during context } \\
\text { switching which leads to slower } \\
\text { switching between threads. }\end{array}$ \\
\hline
\end{tabular}

when one thread is waiting for an I/O. As mentioned above, however, asynchronous $\mathrm{I} / \mathrm{O}$ is not used in the current version of PEMS and direct $\mathrm{I} / \mathrm{O}$ is used only for swapping contexts of virtual processors between memory and disks.

\subsubsection{Kernel Level Threads Versus User Level Threads}

In our implementation each virtual processor is simulated as a user space thread. We could have used kernel space threads. Table 4.6.4 summarizes the advantages and disadvantages in using one over the other.

We decided to use the user space threads to shorten development time and reduce the complexity of the system; they are easier to manage and synchronize, switching between threads is faster and coding and debugging is easier. We chose the GNU pth thread library for our implementation. This library is portable, has a POSIX pthread interface as well as has its own specific interface. 


\section{Chapter 5}

\section{Experiments}

The main objective of our present work has been to see whether the simulation technique as proposed in $[12,30]$ is practical. At this stage of PEMS development, our experiments focus primarily on scalability of performance when the problem size and number of processors are varied.

We describe a series of experiments which test the implementation and the theory for their correctness, efficiency, linearity and scalability. These experiments have been performed using sorting and list ranking as our problems. We have also tested EM_Alltoallv as our most sophisticated and most I/O intensive primitive to measure PEMS overhead without any algorithms involved. We have tested the system in single processor mode and multiple processor (machine) mode. We also look at the effects of disk striping. In general, PEMS seems to have a very good behavior and from our preliminary experimental results we can conclude that simulating a coarse grained parallel algorithm as parallel external memory algorithm is an effective technique both in practice as well as in theory. 


\subsection{Experimental Setup}

All single processor experiments were performed on a machine with configuration $A$ as listed in Appendix A.1.

Multiple processor experiments with parallel sample sort, including linearity, scalability and comparison with TPIE [48] and STXXL [16] were performed on a cluster of Linux machines with hardware and software configuration B as listed in Appendix A.2. This cluster was dedicated to our tests.

List ranking experiments and multiple machine experiments with EM_Alltoallv were performed on a cluster of Linux machines with hardware and software configuration $C$ as listed in Appendix A. ${ }^{1}$

In order to minimize effects of the operating system swap mechanism, it has been disabled in most of the tests. Operating system swap was on for the list ranking experiment.

\subsection{Testing EM_Alltoallv}

The objective of this experiment is to see how PEMS performs when no user program is involved. We choose EM_Alltoallv for this test since this EM primitive is the most $\mathrm{I} / \mathrm{O}$ intensive and most complex primitive in PEMS. It is also the only primitive that contains the implementation of balanced routing algorithm (see Sections 3.3 and 4.5.2). So it can be a representative of the worst-case overhead of the PEMS system. The reader should note that not all communications in a user program

\footnotetext{
${ }^{1}$ Configuration $A$ is a development machine. Configuration $B$ is a large parallel system as part of HPCVL consortium [28], but it was unfortunately not available when we were doing list ranking experiments since it was getting upgraded. Configuration $C$ is the cluster within our school's undergraduate lab.
} 


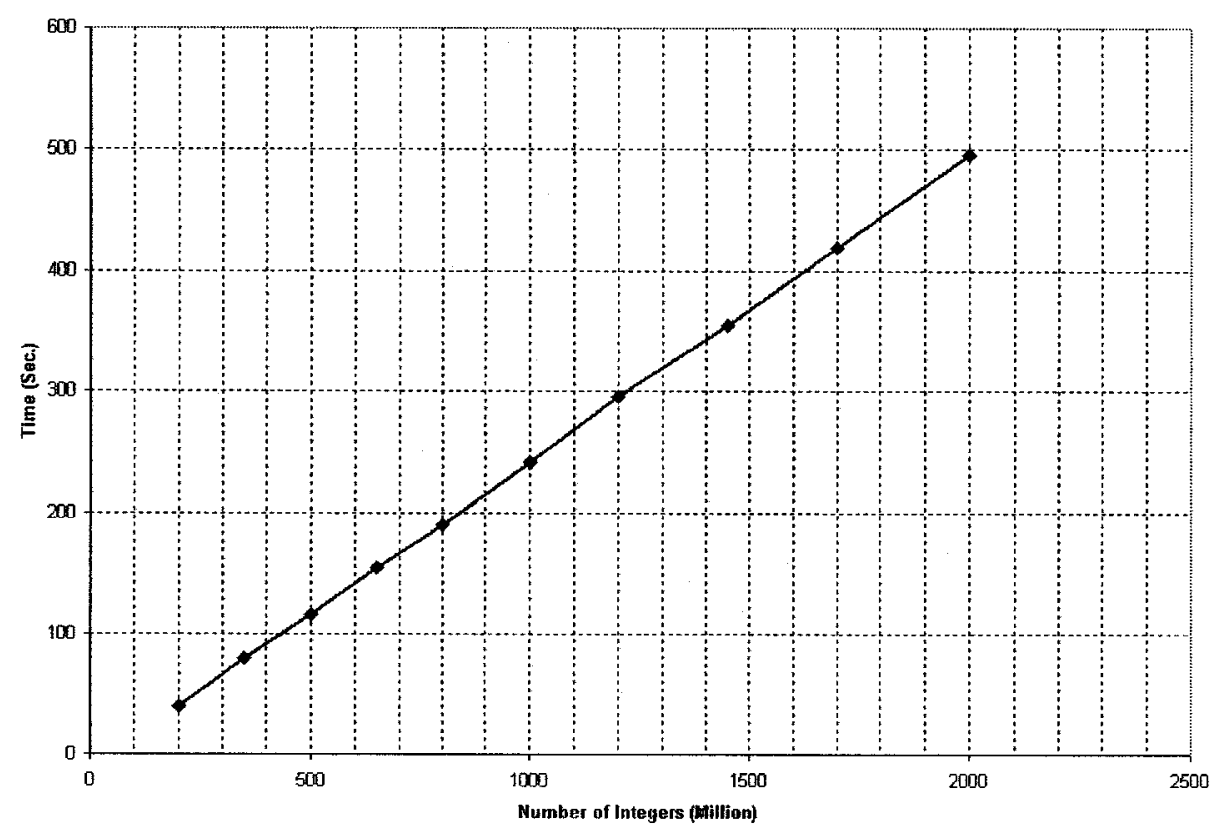

Figure 5.1: Wall clock timings for EM_Alltoallv on a single processor. The $\mathrm{X}$-axis is the problem size in millions of integers. The $\mathrm{Y}$-axis is wall clock time in seconds.

requires EM_Alltoallv. In all of these experiments each processor sends and receives $h=\frac{N}{v}$ number of integers.

\subsubsection{Single Processor Experiments with EM_Alltoallv}

We performed two experiments in the single processor mode. In the first one we time EM_Alltoallv and we vary the total data size $(N)$. As we use a fixed amount of internal memory (half of the physical memory), we have to increase the number of virtual processors as we increase the data size to accommodate the data. Using half of the physical memory each virtual processor can handle almost 125 million integers (on machine configuration A). Figure 5.1 shows the results of this experiment.

For the second experiment we used a fixed size data and varied the number of 


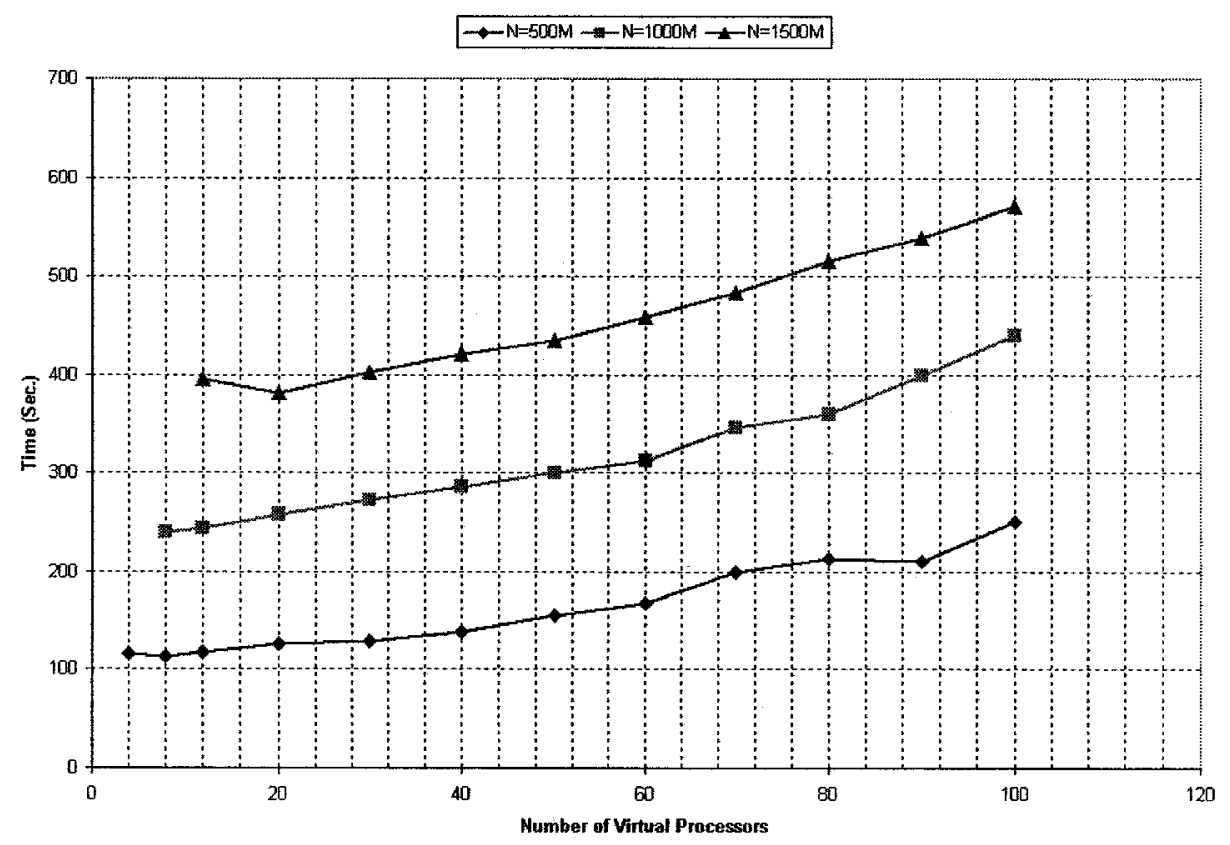

Figure 5.2: Wall clock timings of EM_Alltoallv for data sizes of 500, 1000 and 1500 million integers. The $\mathrm{X}$-axis is the number of virtual processors. The $\mathrm{Y}$-axis is wall clock time in seconds.

virtual processors. This experiment was repeated for three different data sizes. This experiment reveals the overhead of using more threads. The resulting graph is depicted in Figure 5.2. Increasing the number of virtual processors from 8 to 100 (12.5 times) for $N=500$ million integers, increases the wall clock time from 113 Sec. to 251 Sec. (2.2 times). This shows that PEMS process management system does not create an unacceptable overhead. However, minimizing this overhead is crucial to PEMS as the ability to increase $v$ allows PEMS to adapt to larger problems. This is an area for future work. 


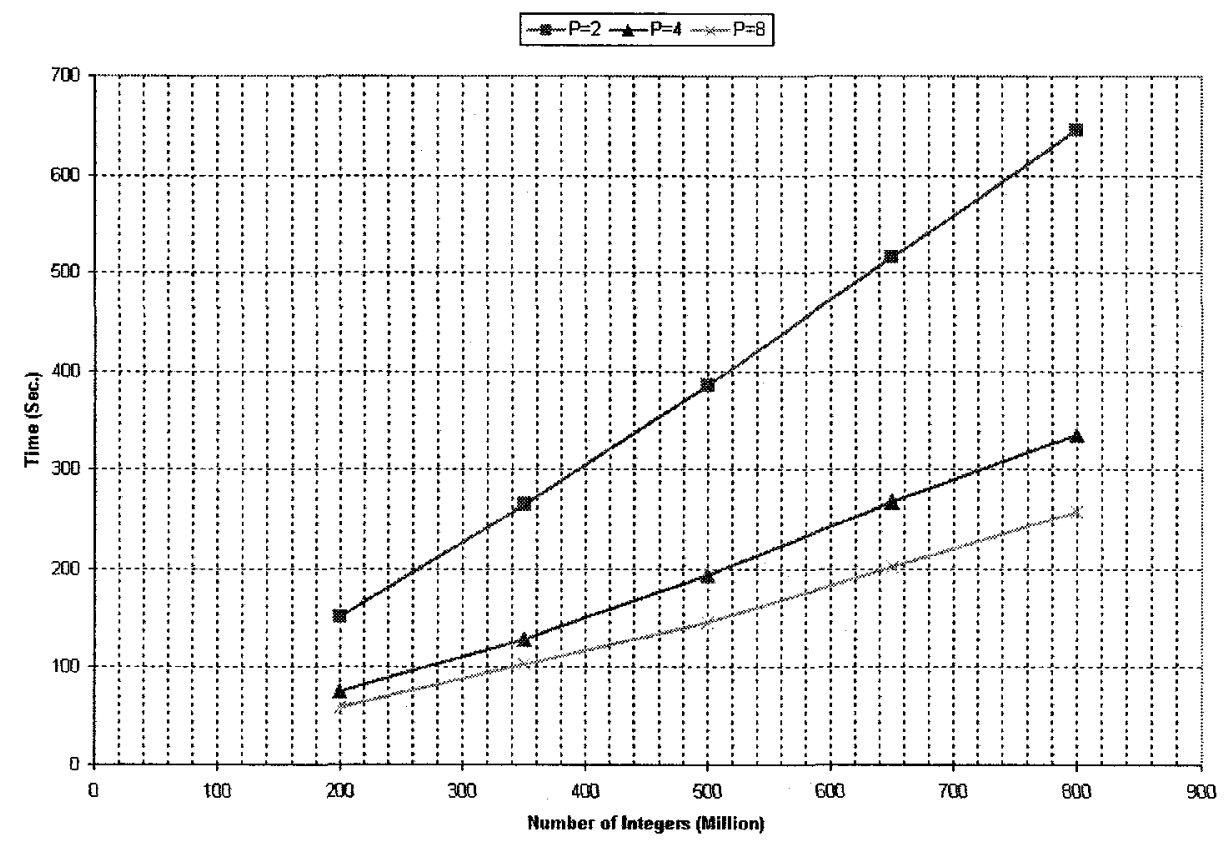

Figure 5.3: Wall clock timings of EM_Alltoallv for $p=2,4$ and 8. The $\mathrm{X}$-axis is the problem size in millions of integers. The $\mathrm{Y}$-axis is wall clock time in seconds.

\subsubsection{Multiple Processor Tests}

We performed one experiment similar to the experiment in single processor mode but here we are only interested in effects of number of real processors. In this experiment we timed EM_Alltoallv and varied the data size. This is done with different number of processors. As it is shown in Figure 5.3 increasing the number of real processors reduces the wall clock time of exchanging data via EM_Alltoallv. In this experiment we kept the number of virtual processors to the minimum needed to handle the data.

Figure 5.4 depicts the normalized version of the graph in Figure 5.3. By normalizing we mean, the wall clock time is divided by problem size. So in this graph Y-axis is the wall clock time per one integer. This figure shows that the overhead is relatively small and the time spent to exchange one integer is almost constant while the data 


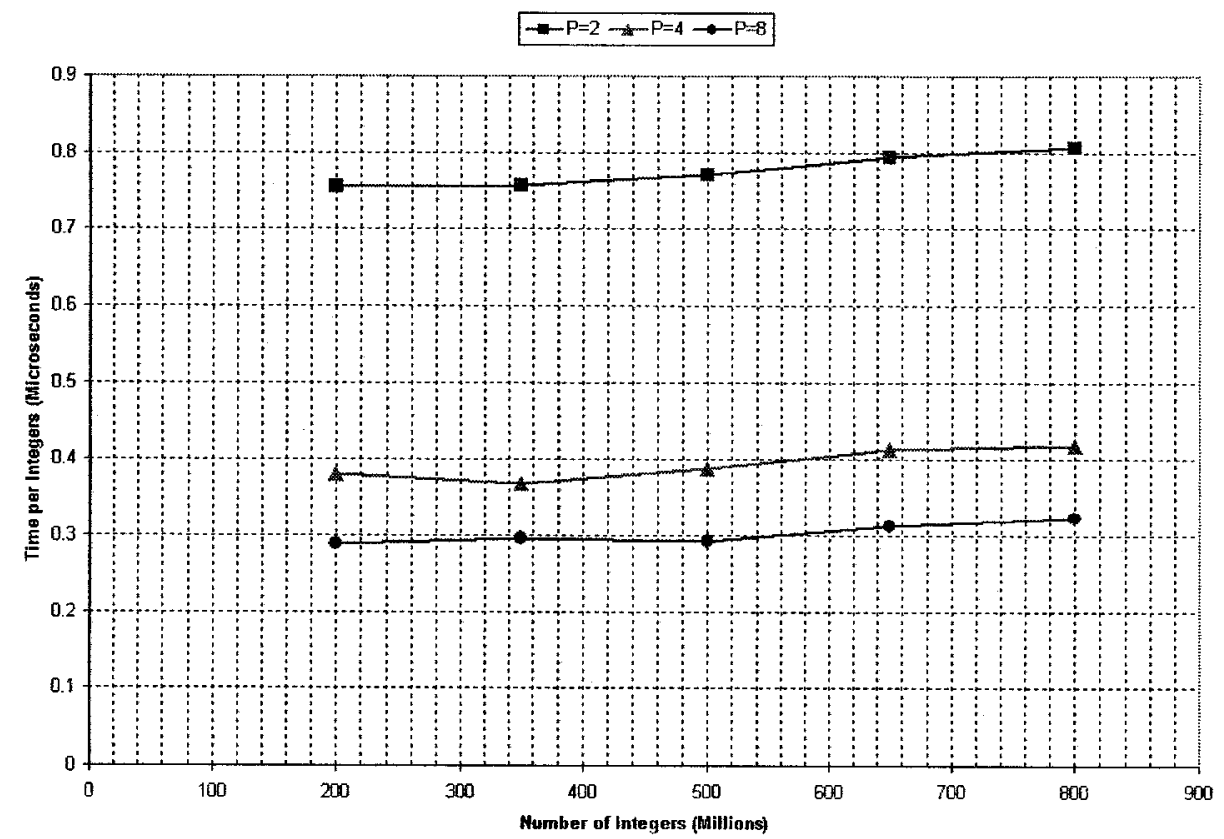

Figure 5.4: Normalized wall clock timings of EM_Alltoallv for data sizes of 200, 500 and 800 million integers.

size increases. This is more evident when there are more real processors.

\subsection{Parallel Sample Sort}

We use parallel sample sort as the first application example to benchmark PEMS. Sorting is a fundamental problem in computer science and has been a hot topic of study in external memory as well as in parallel computations. In our experiments we have simulated parallel sample sort [42] as an external memory algorithm. Algorithm 5.1 is an outline of parallel sample sort as described in [42]. We borrowed the MPI code from Dehne [53] and modified it to suite our objectives. 
Algorithm 5.1 Parallel sample sort algorithm on $v$ virtual processor (from [42]).

INPUT: $N$ random integers evenly distributed among $v$ processors.

OUTPUT: $N$ sorted integers distributed among $v$ processors, none of them stores more than $\frac{2 N}{v}$ integers.

1. Each processor sorts its local data.

2. Each processor chooses $v$ equally spaced elements (splitters).

3. All processors send their $v$ splitters to processor $v_{0}$.

4. $v_{0}$ sorts all $v^{2}$ splitters and picks up each $v^{\text {th }}$ splitter.

5. $v_{0}$ broadcasts the $v-1$ global splitters to all processors.

6. Each processor partitions its sorted data with respect to global splitters and then send the $i^{\text {th }}$ partition to processor $v_{i}$.

7. Each processor sorts its received data.

\subsubsection{Experimental Results with Parallel Sample Sort}

We have performed a series of experiments with parallel sample sort in single processor mode and multiple processor mode. Test data was generated using the $\mathrm{C}$ or $\mathrm{C}++$ standard random generator or the package specific random generator method. We do not use any special integer sorting techniques in the parallel sample sort algorithm or in other test programs. The results of these experiments are presented in the following subsections.

\subsubsection{Single Processor Experiments}

We first ran the parallel sample sort as a sequential external memory program. Figure 5.5 summarizes the timing results. In this experiment we compared a RAID 0 partition 


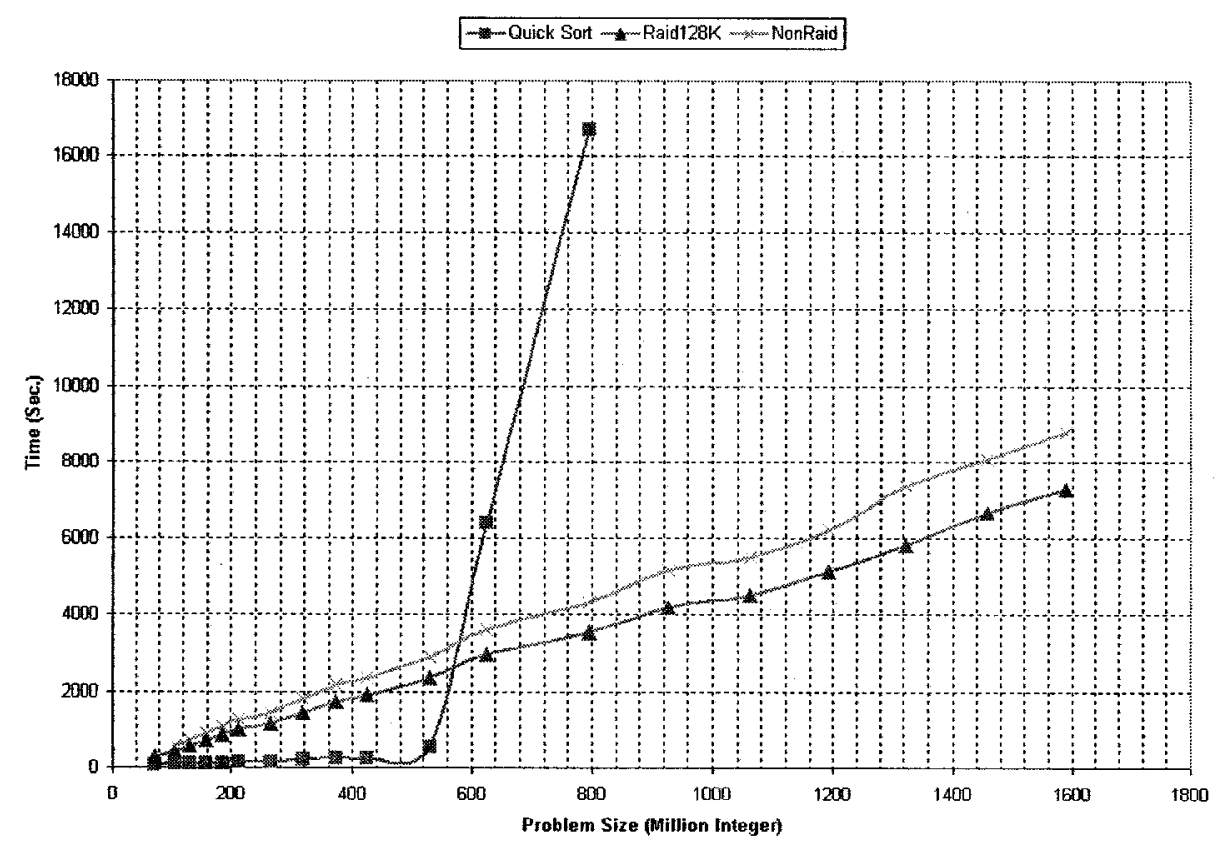

Figure 5.5: Single processor results, $\mathrm{X}$-axis is the problem size in millions of integers. The Y-axis is the wall clock time in seconds.

with a regular disk partition. The RAID 0 partition is a software RAID 0 or striping RAID with two partitions across two disks which is managed by the Linux kernel. Since the transfer rate of the RAID partition was almost $60 \%$ more than that of NONRAID partition as reported by the Linux hdparm utility, we anticipated around $60 \%$ increase in performance. Our timings show that the simulation done using the RAID 0 partition performs only $20 \%$ faster in comparison to the regular disk partition. One reason for this difference is that the hdparm utility does not provide an accurate measure of the disk speeds. We have observed that it is sensitive to the physical location of the RAID partition across the disks.

Figure 5.5 also shows single processor timings of the $\mathrm{C}$ standard library qsort function for comparison. Unlike our PEMS experiments, qsort's timing has been done with the operating system's swap turned on so that it can use virtual memory. 


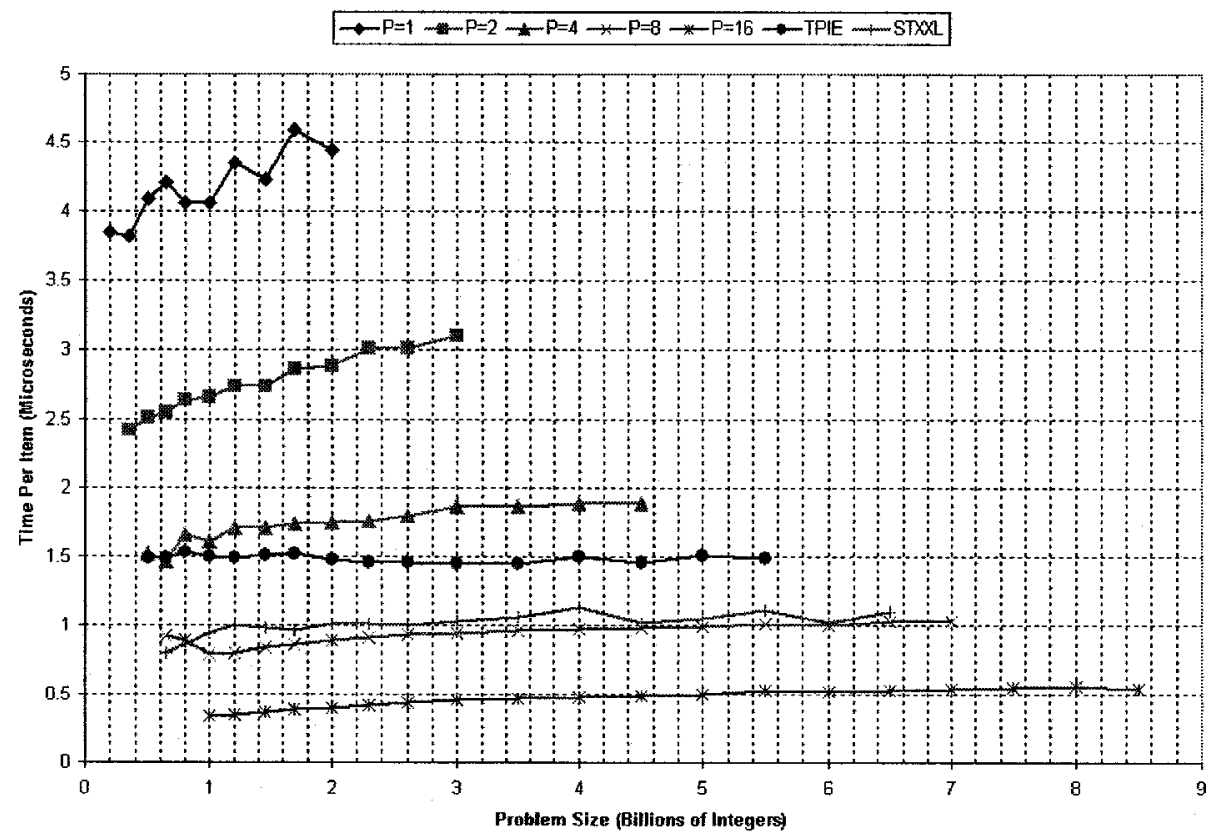

Figure 5.6: Normalized wall clock timings for sorting. The $\mathrm{X}$-axis is the problem size in billions of integers. The Y-axis is wall clock time per data item in microseconds.

As expected qsort execution time increases exponentially as data size exceeds the size of main memory.

\subsubsection{Multiple Processor Experiments}

In this subsection we present the results of multiple processor experiments with parallel sample sort. For comparison, we include timings from TPIE's (see Section 2.4.1) test_sort and STXXL's (see Section 2.4.3) test_sort1. We have slightly modified TPIE's test_sort and STXXL's test_sort1 functions to restrict them to one round of sorting (with no extra tests). All of the test programs use $128 \mathrm{MB}^{2}$ of RAM for sorting.

The record size is 4 bytes and timing includes the data generation, but none of the test programs create a separate output file. PEMS uses $128 \mathrm{MB}$ of memory but

\footnotetext{
${ }^{2} \mathrm{MB}$ and $\mathrm{GB}$ (megabytes and gigabytes) are respectively $2^{20}$ and $2^{30}$ bytes.
} 


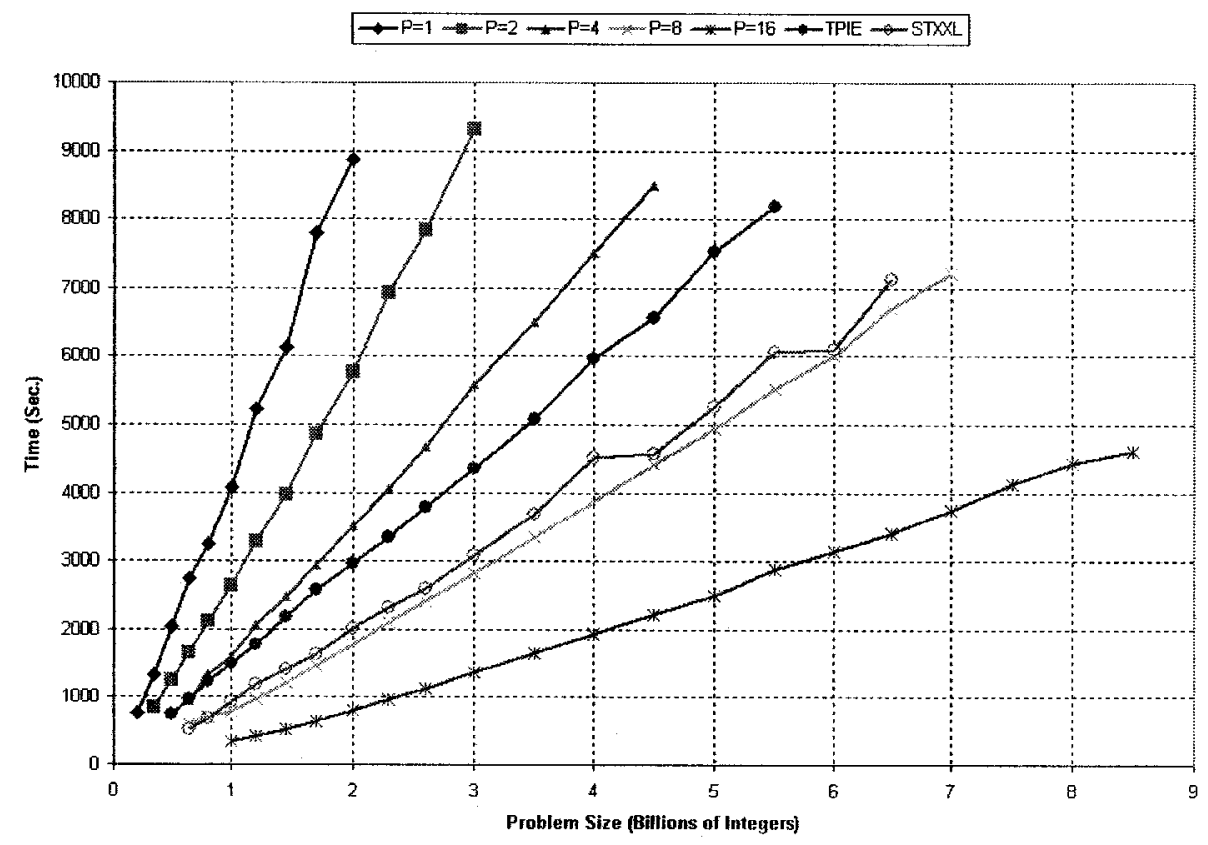

Figure 5.7: Wall clock timings for sorting. The $\mathrm{X}$-axis is the problem size in billions of integers. The $\mathrm{Y}$-axis is wall clock time in seconds.

$64 \mathrm{MB}$ of this memory is used as shared buffer for communication, and only $64 \mathrm{MB}$ is used for sorting. (In PEMS tests, we have disabled the extra memory so that the operating system cannot use it for caching. ${ }^{3}$ )

Figure 5.6 shows the normalized running times for PEMS sample sort on 1, 2, 4,

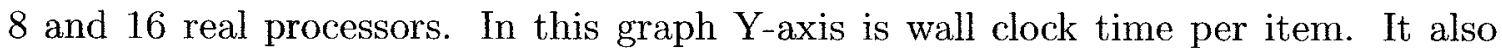
includes normalized running time of TPIE and STXXL external memory sort test programs on a single processor. We include the TPIE and STXXL results only as general reference points that highlight room for improvement in our single processor results. The reader should not draw conclusions about the relative running times of TPIE and STXXL sort, for instance, as we have not ensured that this is a fair

\footnotetext{
${ }^{3}$ This was done by allocating and initializing the extra memory in the sort program without using this extra allocated memory for any computation.
} 


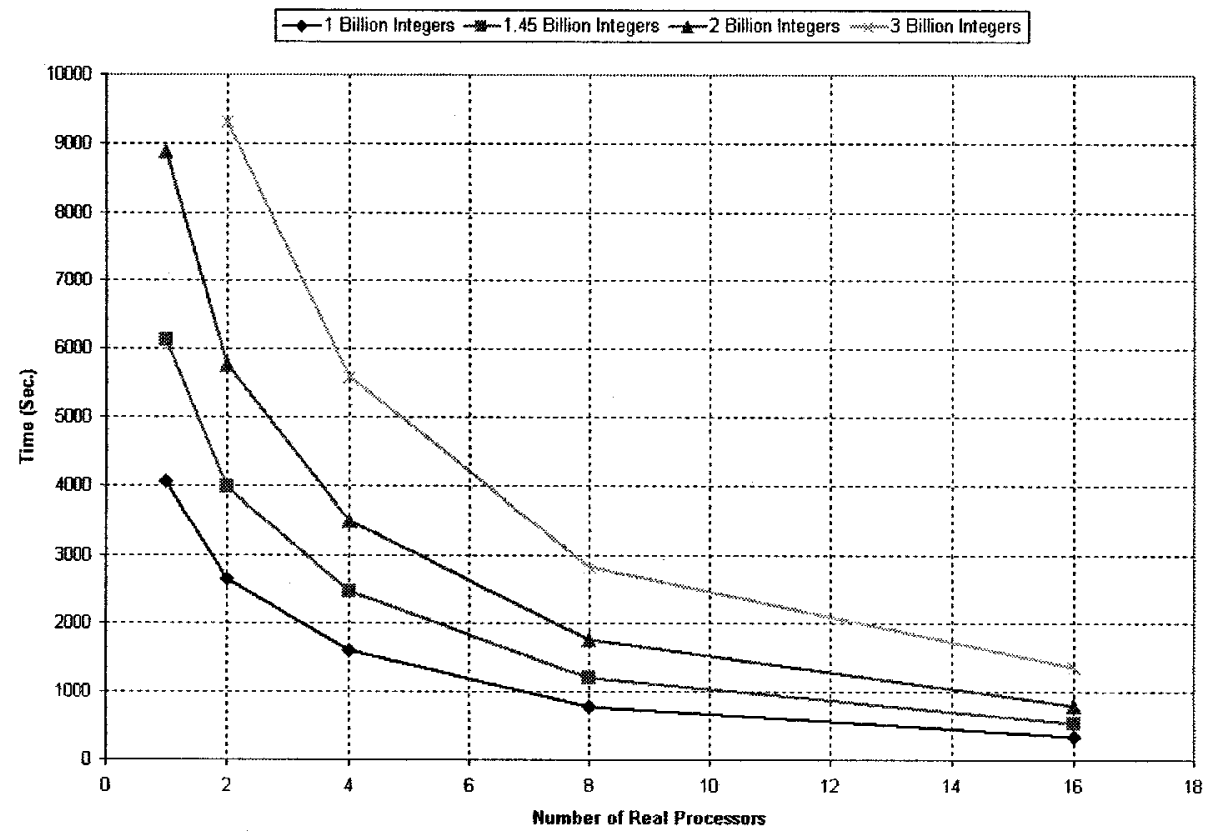

Figure 5.8: Speedup of PEMS Sample Sort.

comparison.

The TPIE and STXXL sort programs are 3 to 5 times faster than our single processor sort with this version of PEMS, but as more real processors are added, PEMS becomes faster. At 7 billion integers, the running time of PEMS with 16 processors reduces by $65 \%, 53 \%$, and $48 \%$, in comparison to TPIE, STXXL and PEMS with 8 processors, respectively. These may be more evident in Figure 5.7 which depicts the non-normalized timings of the experiments.

As Figure 5.6 shows the normalized PEMS running times have a very small growth when problem size increases and the number of real processors is fixed. This is specially true when more than two real processors are in the system. In addition, Figure 5.8 shows how the running time of PEMS sample sort decreases as the number of real processors increases. In that graph, if the number of real processors is doubled, 
Algorithm 5.2 Simple Randomized Parallel List Ranking (from [14])

INPUT: A linked list distributed evenly among $v$ processors $\left(\frac{N}{v}\right.$ nodes per processor).

OUTPUT: The same linked list with computed rank for each node saved in the data structure of that node.

1. Each processor $v_{i}$ selects each $x$ in its local list as a pivot with probability $\frac{1}{v}$. So with high probability $O\left(\frac{N}{v}\right)$ nodes are selected as the pivot set.

2. All processors collectively compute a "partial rank": the distance between a node $x$ and the nearest point in the pivot set for all $x \in S$ using Algorithm 5.3.

3. Using Alltoallv the distances computed in Step 2 for pivot set is sent to all processors.

4. Using the data received in Step 3, each processor $v_{i}$ solves the list ranking problem for nodes stored in $v_{i}$ sequentially.

the running time is almost halved. For example when $N=3$ billion integers, the timing for $p=8$ and $p=16$ are respectively 2822 and 1369 seconds.

\subsection{Randomized Parallel List Ranking}

Given a linked list of nodes, the list ranking problem is the computation of the distance of each node from the last node or tail. The distance between two nodes can be defined as the number of links between the two nodes. The sequential version of this problem has a simple linear time solution. But as there is no assumption on the organization of the nodes in the memory, it has a highly random memory access pattern. This behavior makes this problem a hard problem when locality in reference should be exploited. 
There are a few randomized and deterministic parallel list ranking algorithms in the literature. We use the randomized parallel list ranking algorithm proposed by Dehne and Song [14] to test our external memory implementation. This is a CGM algorithm where the input is a set $S$ containing $N$ nodes. Each processor contains $\frac{N}{v}$ nodes. The output is the same set of nodes with their ranks computed and saved within each node. Algorithm 5.2 outlines the main steps of this algorithm. Step 2 of this algorithm is performed using the pointer jumping technique shown as Algorithm 5.3. In this algorithm each iteration of the while loop involves two Alltoallv communication rounds. For instance, in the very first iteration, each processor requests values for next(next $(x))$ and partial_rank(next $(x))$ for those $x$ in its local memory, then in the second round each processor receives its requested results.

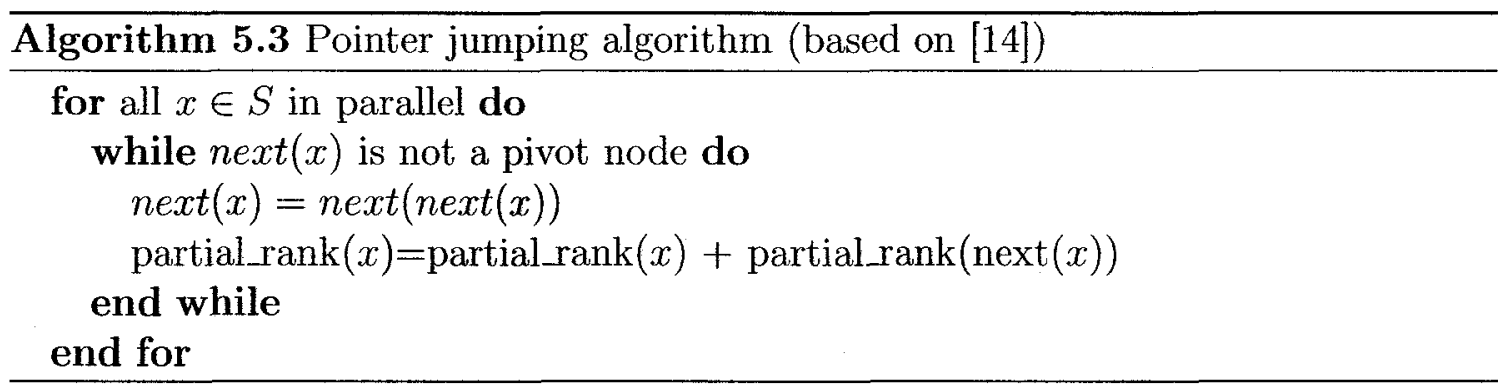

Theorem 5.1 [14] Algorithm 5.2 requires with high probability $O(\log v+\log \log N)$ communication rounds (w.h.p, $h=O\left(\frac{N}{v}\right)$ ) and $O\left(\frac{N}{v}\right)$ local computation.

\subsubsection{Experimental Results with List Ranking}

We implemented Algorithm 5.2 as a standalone MPI program ${ }^{4}$. In this implementation we assume that the set of nodes is an array and nodes are distributed among the

\footnotetext{
${ }^{4}$ We examined the implementation of this algorithm in CGMGRAPH/CGMLIB (http://www.scs.carleton.ca/ $\sim \mathrm{cgm}$ ), but decided to implement our own since that is not a standalone MPI implementation.
} 


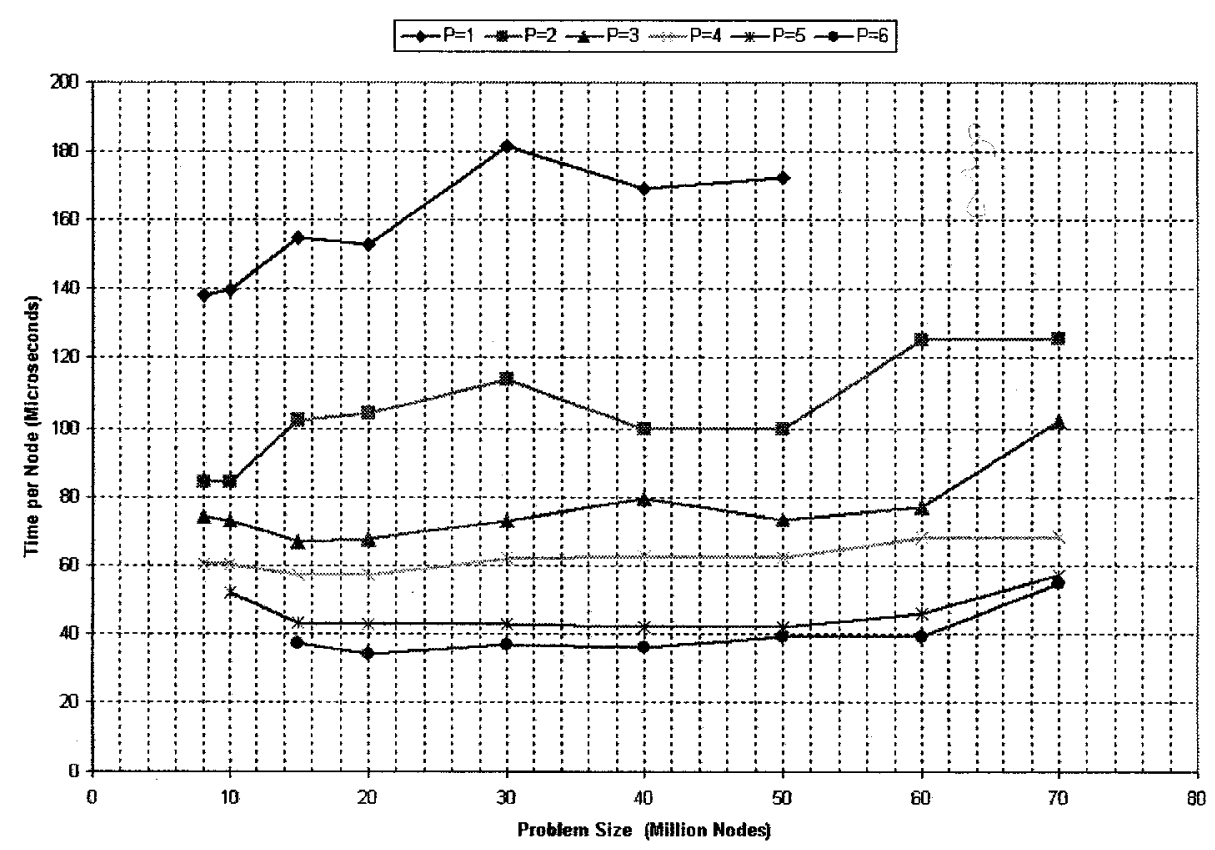

Figure 5.9: Normalized wall clock timing of list ranking on 1,2,3,4,5 and 6 real processors.

processors.

We then converted this program into an EM program using PEMS. This code was timed on the Zeta cluster with configuration $C$ as listed in Appendix A.3. In these experiments the amount of internal memory on each real processor was limited to half of the physical memory. Note that in this implementation each node representation requires 28 bytes of data. So just keeping a relatively small linked list in memory requires a relatively huge amount of memory. (As an example a list of 50 million nodes needs almost 1.3GB of memory.) Our timings include the time needed to read the input file and the time needed to save the output list.

The linked list for this experiment was generated randomly by another sequential program and the generated linked list nodes are randomly distributed among the processors. At the end of execution of the program each processor saves its portion 


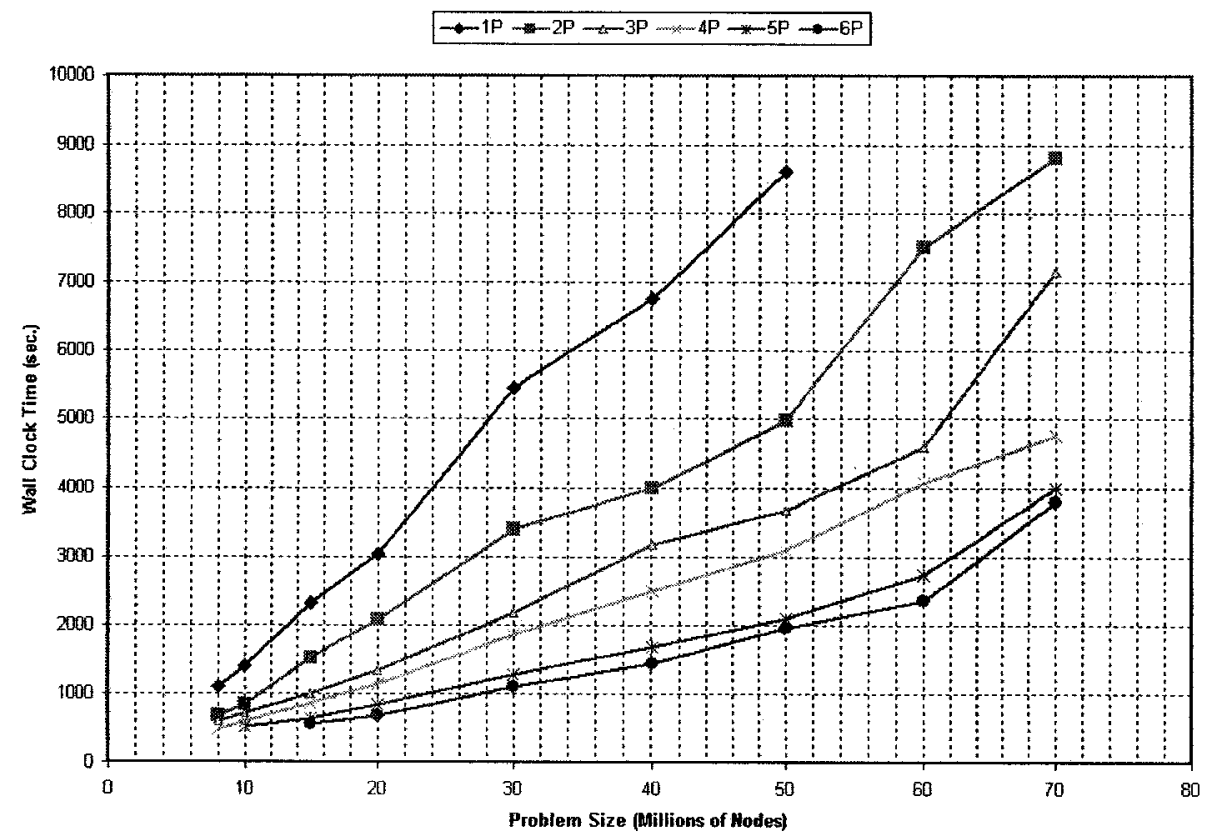

Figure 5.10: Wall clock timing of list ranking on $1,2,3,4,5$ and 6 real processors.

of the linked list (ranks are saved in the node information) in a local file. Figure 5.9 shows that the running times of our EM list ranking program decreases as number of real processors increases. As we saw in Theorem 5.1 the complexity of this algorithm involves a $\log v$ term. This logarithmic term brings $N$ back in since we have to increase $v$ in order to accommodate larger data sets. The graphs in Figure 5.9 indicate this behavior. This is more evident in Figure 5.10 which is the non-normalized timing of the list ranking experiment.

Figure 5.11 shows speedup of the EM list ranking. Here the speedup is not as attractive as in the sort problem. But this is intrinsic in the list ranking problem and its present solutions, so even in a pure parallel environment it is not possible to achieve linear speedup. 


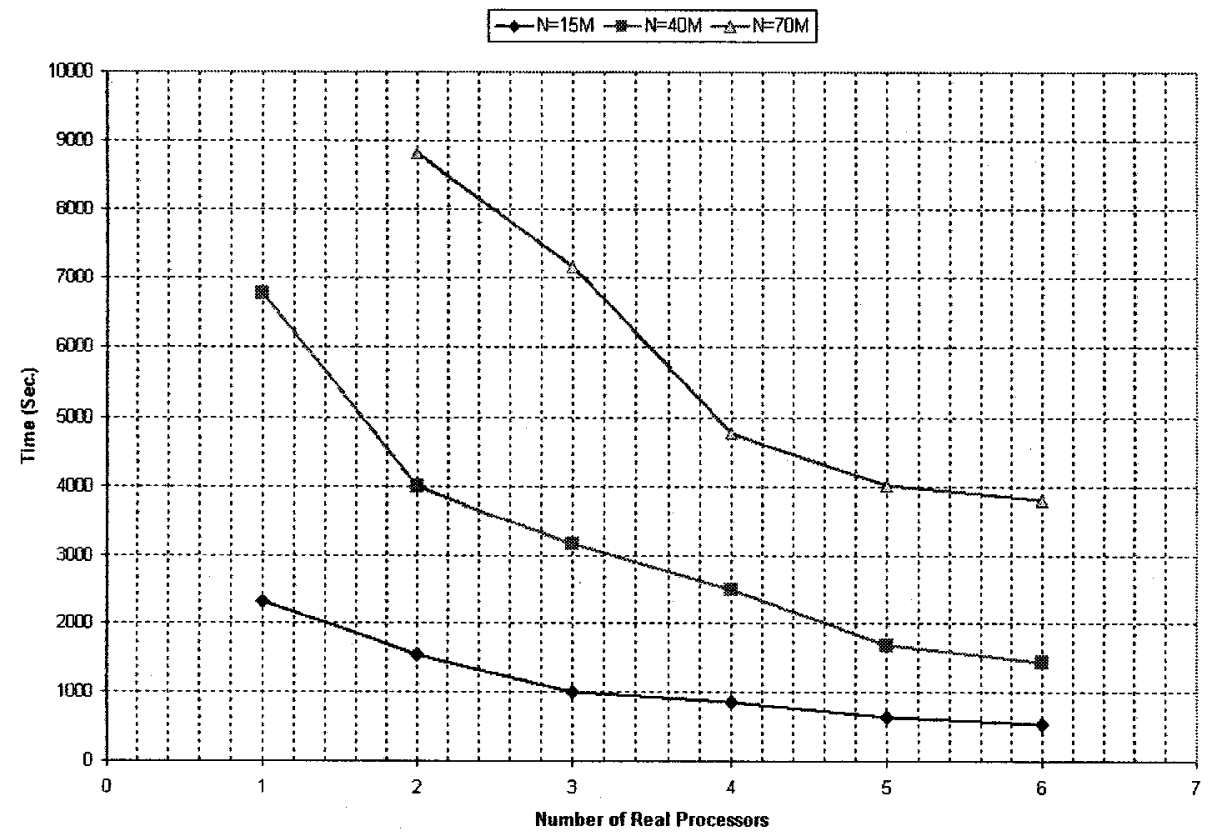

Figure 5.11: Speedup of PEMS list ranking as number of real processors increase. The $\mathrm{X}$-axis is the number of real processors. The $\mathrm{Y}$-axis is wall clock time in seconds.

\subsection{Conclusions}

These experiments show several important properties of this approach. First of all the methodology is practical. The running times with a fixed number of processors is what the theory predicted [12]. Secondly, increasing the number of real processors decreases the running time in a predictable way. Increasing the number of real machines also increases the aggregate amount of RAM and the number of disks. We believe that the good speedups in parallel running time in our tests may be primarily due to this disk parallelism. 


\section{Chapter 6}

\section{Concluding Remarks And Future}

\section{Work}

PEMS is a runtime library for creating parallel external memory programs from implementations of BSP-like coarse grained parallel algorithms. The primary application area is problems that require processing of massive amounts of data. In [12], a large number of suitable parallel algorithms are identified for useful problems in graphs and computational geometry. PEMS promises to facilitate parallel EM implementations of these algorithms.

Our experiments show several important properties of the PEMS approach. First of all the methodology is practical. The running times with a fixed number of processors is what the theory predicted [12]. Secondly, increasing the number of real processors decreases the running time in a predictable way. The ability to exploit parallel machine resources such as disks gives the ability to handle extremely large data sets on practical architectures such as a network of workstations. On such a system, one can inexpensively add computational power, RAM, disks, and bandwidth 
between RAM and disk by adding machines to a network. Using coarse grained parallel algorithms and PEMS, our experiments in this work suggest that one can take advantage of all of these resources, as well as adapting the theory of coarse grained parallel algorithms to the reality of a smaller number of real processors, each with a disk system. We believe that our experiments with sorting, for instance, showed good speedups in parallel running time primarily due to disk parallelism. More computationally intensive applications may be able to also make use of the additional computational power.

This brings us to several suggestions for further work.

(1) PEMS algorithms have the disadvantage that they do more $\mathrm{I} / \mathrm{O}$, by a constant factor, than a conventional single processor EM algorithm due to the need to swap the contexts of virtual processors between RAM and disk. Our experiments suggest that this can be offset by the scalability of I/O bandwidth in our model. However, since $\mathrm{I} / \mathrm{O}$ is so prevalent in PEMS we expect that improving the low level $\mathrm{I} / \mathrm{O}$ performance may make a significant improvement in running times. To this end we suggest that the use of asynchronous (no-wait) I/O should be investigated in PEMS. This would allow the overlapping of computation and $\mathrm{I} / \mathrm{O}$ and the use of multiple independent parallel disks on each real processor. But this requires a redesign of the PEMS Memory Management and Process Management layers to enable PEMS to have at least two active threads on each real processor at any time. Then while one thread is waiting for $\mathrm{I} / \mathrm{O}$ the other thread performs computation.

(2) We noticed that STXXL [16] has a well designed and efficient asynchronous parallel disk I/O layer which can be used without calling its higher level functions. The possible use of STXXL's asynchronous parallel disk I/O layer in the PEMS disk 
I/O subsystem may be investigated.

(3) As most of PEMS I/O operations are linear reading/writing or streaming I/O, it may be possible to use STXXL streaming functions or other streaming I/O libraries instead of low level I/O functions.

(4) It is possible to further investigate the use of kernel threads in the asynchronous sending and receiving of communication traffic between virtual processors. Using this technique, it will also be possible to send and receive smaller blocks of messages and hence reduce the size of buffers needed for communication.

(5) An important limitation of PEMS is its internal disk usage. It needs $\frac{v}{p} \times \mu_{d}$ bytes of disk space for swapping of data on each real processor. Here $\mu_{d}$ represents the size of the data memory of each virtual processor. It also reserves $2 \frac{v}{p} \times \mu_{d}$ bytes of disk space for communication on each real processor. Some effort should be put towards reducing these requirements.

(6) With multiple core CPUs becoming a commodity, adjustments should be considered for PEMS to take full advantage of symmetric multiprocessor (SMP) machines. This could involve the use of kernel level threads to create virtual processors instead of user level threads.

(7) It would be interesting to get an idea of when network bandwidth limits the scalability of a PEMS application.

(8) In order to study the behavior of PEMS when simulating different algorithms more examples should be implemented. In [12] the EM-BSP I/O complexity of more than 8 classes of algorithms including many graph and computational geometry algorithms are derived. These algorithms may be considered for more experimental studies. The challenge however, may be the fact that many of these algorithms do 
not have a standalone MPI implementation or even may not have been used in practice at all. 


\section{Appendices}




\section{Appendix A}

\section{System Configurations for Experiments}

\section{A.1 Machine Configuration A for Single Processor Tests}

- CPU: AMD Opteron $2.4 \mathrm{GHz}$ with $2 \mathrm{~GB}$ of RAM.

- HD: 3 Hard Disks each with at least 30GB of free space.

- Two Partitions across two disks configured as software RAID 0.

- Hard Disk Bandwith: 71MB/Sec. RAID Bandwith: 112 MB/Sec.

- File System: EXT3

- OS: GNU/Linux 64bit v2.6.15.

- C Compiler: gec v4.1.1. 


\section{A.2 Cluster Configuration B for Multiple Machine Experiments}

This cluster which is part of HPCVL Lab (www.hpcvl.org) was dedicated to our tests.

- CPU: Intel $2 \times$ Xeon 2.0 GHz with $1.5 \mathrm{~GB}$ of RAM (Only one processor was used in practice).

- HD: 1 Hard Disk each with at least 40GB of free space.

- Hard Disk Bandwith: 45MB/Sec (measured by hdparm utility).

- OS: GNU/Linux 32bit kernel version 2.6.9-42.0.2.ELsmp.

- File System: EXT2

- C Compiler: gcc v 3.4.6.

- Gigabit Ethernet for the communication between machines.

\section{A.3 Cluster Configuration C for Multiple Machine Experiments}

This is the Zeta cluster at the SCS. This cluster was a mixture of different machines. Operating system swap was ON.

- CPU: Intel $2 \times$ Xeon $2.40 \mathrm{GHz}$ (Only one processor was used in practice) or

- CPU: Intel Pentium 4, 2.00GHz or $2.40 \mathrm{GHz}$. 
- RAM: 1GB.

- HD: Standard 7200 RPM hard drive with at least 8GB of free space for EM usage.

- OS: GNU/Linux 32bit kernel version 2.6.18-1.2798.fc6.

- File System: EXT3

- C Compiler: 4.1.2.

- $100 \mathrm{Mb}$ Ethernet for the communication between machines. 


\section{Bibliography}

[1] Aggarwal, A., and Vitter, J. S. The Input/Output complexity of sorting and related problems. Communications of the ACM 31, 9 (1988), 1116-1127.

[2] Anderson, R. J., AND Miller, G. L. A simple randomized parallel algorithm for list-ranking. Information Processing Letters 33 (1990), 269-273.

[3] Arge, L. The buffer tree: A technique for designing batched external data structures. Algorithmica 37, 1 (2003), 1-24.

[4] Arge, L., Procopiuc, O., And Vitter, J. S. Implementing I/O-efficient data structures using tpie. In ESA (2002), R. H. Möhring and R. Raman, Eds., vol. 2461 of Lecture Notes in Computer Science, Springer, pp. 88-100.

[5] Bader, D. A., Helman, D. R., ANd JÁJÁ, J. Practical parallel algorithms for personalized communication and integer sorting. ACM Journal of Experimental Algorithms 1 (1996), 3.

[6] Burns, G., Daoud, R., and Vaigl, J. LAM: An Open Cluster Environment for MPI. In Proc. of Supercomputing Symposium (1994), pp. 379-386. 
[7] Chiang, Y.-J., Goodrich, M. T., Grove, E. F., Tamassia, R., VenGROFF, D. E., AND ViTTER, J. S. External-memory graph algorithms. In Proc. of ACM-SIAM Symp. on Discrete Algorithms (1995), pp. 139-149.

[8] Crauser, And Mehlhorn. LEDA-SM: Extending LEDA to secondary memory. In WAE: International Workshop on Algorithm Engineering (1999), LNCS.

[9] de Berg, M., van Kreveld, M., Overmars, M., and Schwarzkopf, O. Computational Geometry: Algorithms and Applications. Springer-Verlag, Jan. 2000.

[10] Dehne, F., Dittrich, W., And Hutchinson, D. Efficient external memory algorithms by simulating coarse-grained parallel algorithms. In SPAA '97: Proc. of the ninth annual ACM symposium on Parallel algorithms and architectures (New York, NY, USA, 1997), ACM Press, pp. 106-115.

[11] Dehne, F., Fabri, A., and Rau-Chaplin, A. Scalable parallel computational geometry for coarse grained multicomputers. International Journal of Computational Geometry and Applicatios 6 (1996), 379-400.

[12] Dehne, F. K. H. A., Dittrich, W., Hutchinson, D. A., and MaheshWARI, A. Bulk synchronous parallel algorithms for the external memory model. Theory Comput. Syst. 35, 6 (2002), 567-597.

[13] Dehne, F. K. H. A., Hutchinson, D., Maheshwari, A., And Dittrich, W. Reducing $\mathrm{I} / \mathrm{O}$ complexity by simulating coarse grained parallel algorithms. 
In IPPS '99/SPDP '99: Proc. of the 13th International Symposium on Parallel Processing and the 10th Symposium on Parallel and Distributed Processing (Washington, DC, USA, 1999), IEEE Computer Society, pp. 14-20.

[14] Dehne, F. K. H. A., AND Song, S. W. Randomized parallel list ranking for distributed memory multiprocessors. In $A S I A N$ (1996), J. Jaffar and R. H. C. Yap, Eds., vol. 1179 of Lecture Notes in Computer Science, Springer, pp. 1-10.

[15] Dementiev, R. Algorithm Engineering for Large Data Sets. PhD thesis, Fakultät für Informatik, Universität Karlsruhe, Karlsruhe, Germany, 2006.

[16] Dementiev, R., Kettner, L., And SAnders, P. STXXL: Standard template library for XXL data sets. In ESA (2005), G. S. Brodal and S. Leonardi, Eds., vol. 3669 of Lecture Notes in Computer Science, Springer, pp. 640651.

[17] Denning, P. J. Working sets past and present. IEEE Trans. Software Eng. 6, $1(1980), 64-84$.

[18] Engelschall, R. S. Portable multithreading-the signal stack trick for userspace thread creation. In USENIX Annual Technical Conference, General Track (2000), USENIX, pp. 239-250.

[19] Essaïdi, M., Lassous, I. G., And Gustedt, J. Sscrap: An environment for coarse grained algorithms. In IASTED PDCS (2002), S. G. Akl and T. F. Gonzalez, Eds., IASTED/ACTA Press, pp. 393-398.

[20] Forum, M. P. I. MPI: A message-passing interface standard. The International Journal of Supercomputer Applications and High Performance Computing 8, 3/4 (Fall/Winter 1994), 159-416. 
[21] Gabriel, E., FagG, G. E., Bosilca, G., Angskun, T., Dongarra, J. J., Squyres, J. M., Sahay, V., Kambadur, P., Barrett, B., Lumsdaine, A., Castain, R. H., Daniel, D. J., Graham, R. L., and Woodall, T. S. Open MPI: Goals, concept, and design of a next generation MPI implementation. In Proc. of 11th European PVM/MPI Users' Group Meeting (Budapest, Hungary, September 2004), pp. 97-104.

[22] Gebremedhin, A. H., Lassous, I. G., Gustedt, J., and Telle, J. A. Pro: A model for parallel resource-optimal computation. In 16th Annual International Symposium on High Performance Computing Systems and Applications, HPCS (2002), IEEE Computer Society, pp. 106-113.

[23] GNU Pth - The GNU Portable Threads. http://www.gnu.org/software/pth/.

[24] Goodrich, M. T., Tsay, J.-J., Vengroff, D. E., and Vitter, J. S. External-memory computational geometry. In 34th Annual Symposium on Foundations of Computer Science, FOCS (1993), IEEE, pp. 714-723.

[25] Gropp, W., AND LUSK, E. A high-performance MPI implementation on a shared-memory vector supercomputer. Parallel Computing 22, 11 (Jan. 1997), $1513-1526$.

[26] Gropp, W., Lusk, E., Doss, N., ANd SkJellum, A. A high-performance, portable implementation of the MPI message passing interface standard. Parallel Computing 22, 6 (Sept. 1996), 789-828. 
[27] Gustedt, J. Towards realistic implementations of external memory algorithms using a coarse grained paradigm. In ICCSA (2) (2003), V. Kumar, M. L. Gavrilova, C. J. K. Tan, and P. L'Ecuyer, Eds., vol. 2668 of Lecture Notes in Computer Science, Springer, pp. 269-278.

[28] High Performance Computing Virtual Library web site. http://www.hpcvl.org/.

[29] Hume, A. Billing in the large. Handbook of massive data sets (2002), 895-909.

[30] Hutchinson, D. A. Parallel Algorithms in External Memory. PhD thesis, School of Computer Science, Carleton University, 1999.

[31] Hutchinson, D. A., Sanders, P., And Vitter, J. S. Duality between prefetching and queued writing with parallel disks. SIAM Journal on Computing 34, 6 (2005), 1443-1463.

[32] KIM, M. Y. Synchronized disk interleaving. IEEE Trans. Computers 35, 11 (1986), 978-988.

[33] Knuth, D. E. The Art of Computer Programming, Vol. 3 Sorting and Searching. Addison-Wesley, 1998.

[34] Message Passing Interface Standards web site. http://www .mpi-forum .org/.

[35] MPIF, M. P. I. F. MPI-2: Extensions to the Message-Passing Interface. Technical Report, University of Tennessee, Knoxville, 1996. 
[36] Nikseresht, M. R., Hutchinson, D. A., And Maheshwari, A. Experiments with a parallel external memory system (to appear). In 14th IEEE International Conference on High Performance Computing, Goa, India, HiPC (2007).

[37] Nodine, M. H., AND VitTER, J. S. Greed sort: Optimal deterministic sorting on parallel disks. Journal of the ACM 42, 4 (1995), 919-933.

[38] Petersen, L. H. External priority queues in practice. Master's thesis, Department of Computer Science,University of Aarhus, Denmark http://www.daimi.au.dk/ aveng/thesis/, June 2007.

[39] Preparata, F. P., And Shamos, M. I. Computational Geometry: An Introduction. Springer-Verlag, 1985.

[40] Rau-Chaplin, A. On Parallel Data Structures and Parallel Geometric Applications for Multicomputers. PhD thesis, School of Computer Science, Carleton University, 1992.

[41] Salem, K., and Garcia-Molina, H. Disk striping. In Proc. of the Second International Conference on Data Engineering, ICDE (1986), IEEE Computer Society, pp. 336-342.

[42] Shi, H., AND SChaEffer, J. Parallel sorting by regular sampling. Journal of Parallel and Distributed Computing 14 (1992), 361-372.

[43] Sibeyn, J. From parallel to external list ranking. Tech. Rep. MPI-I-97-1021, Max-Planck-Institut für Informatik, Saarbrücken, Germany, 1997. 
[44] Sibeyn, J., and Kaufmann, M. Bsp-like external-memory computation. In Proc. 3rd Italian Conference on Algorithms and Complexity, Lecture Notes in Computer Science, LNCS 1203 (1997), Springer, pp. 229-240.

[45] Sibeyn, J. F. External connected components. In 9th Scandinavian Workshop on Algorithm Theory, SWAT (2004), T. Hagerup and J. Katajainen, Eds., vol. 3111 of Lecture Notes in Computer Science, Springer, pp. 468-479.

[46] Squyres, J. M., And Lumsdaine, A. A Component Architecture for LAM/MPI. In Proc., 10th European PVM/MPI Users' Group Meeting (Venice, Italy, September / October 2003), no. 2840 in Lecture Notes in Computer Science, Springer-Verlag, pp. 379-387.

[47] Valinnt, L. G. A bridging model for parallel computation. Communications of the ACM 33, 8 (August 1990), 103-111.

[48] Vengroff, D. E. A transparent parallel I/O environment. In Proc. 1994 DAGS Symposium on Parallel Computation (1994).

[49] VitTer, J. S. External memory algorithms and data structures. ACM Comput. Surv. 33, 2 (2001), 209-271.

[50] Vitter, J. S., And Hutchinson, D. A. Distribution sort with randomized cycling. Journal of the ACM 53, 4 (2006), 656680.

[51] VitTer, J. S., AND Shriver, E. A. M. Algorithms for parallel memory, I: Two-level memories. Algorithmica 12, 2-3 (1994), 110-147.

[52] Vitter, J. S., AND Shriver, E. A. M. Algorithms for parallel memory, II: Hierarchical multilevel memories. Algorithmica 12, 2-3 (1994), 148-169. 
[53] Yogaratnam, K. Simulation of CGM Algorithms as EM-CGM Algorithms. Honours project report, School of Computer Science, Carleton University, Ottawa, 1998. 